\title{
EXTREMES OF AUTOREGRESSIVE THRESHOLD PROCESSES
}

\author{
CLAUDIA BRACHNER, ${ }^{*}$ Allianz Investment Management SE \\ VICKY FASEN, ${ }^{* *}$ Technische Universität München \\ ALEXANDER LINDNER, ${ }^{* * *}$ Technische Universität Braunschweig
}

\begin{abstract}
In this paper we study the tail and the extremal behaviors of stationary solutions of threshold autoregressive (TAR) models. It is shown that a regularly varying noise sequence leads in general to only an O-regularly varying tail of the stationary solution. Under further conditions on the partition, it is shown however that $\operatorname{TAR}(S, 1)$ models of order 1 with $S$ regimes have regularly varying tails, provided that the noise sequence is regularly varying. In these cases, the finite-dimensional distribution of the stationary solution is even multivariate regularly varying and its extremal behavior is studied via point process convergence. In particular, a TAR model with regularly varying noise can exhibit extremal clusters. This is in contrast to TAR models with noise in the maximum domain of attraction of the Gumbel distribution and which is either subexponential or in $\mathcal{L}(\gamma)$ with $\gamma>0$. In this case it turns out that the tail of the stationary solution behaves like a constant times that of the noise sequence, regardless of the order and the specific partition of the TAR model, and that the process cannot exhibit clusters on high levels.
\end{abstract}

Keywords: Ergodic; exponential tail; extreme value theory; O-regular variation; point process; regular variation; SETAR process; subexponential distribution; tail behavior; TAR process

2000 Mathematics Subject Classification: Primary 60G70

Secondary 60G10; 60G55

\section{Introduction}

A (self-exciting) threshold autoregressive ((SE)TAR) model of order $q$ with $S$ regimes is a piecewise $\operatorname{AR}(q)$ process with different regimes, where the current regime depends on the size of the past observations. More precisely, we will consider the following model: let $\left(Z_{k}\right)_{k \in \mathbb{N}_{0}}$ be an independent and identically distributed (i.i.d.) noise sequence, let $q, p, S, d_{1}, \ldots, d_{p} \in \mathbb{N}$ with $d_{1}<\cdots<d_{p}$, let $\left\{J_{i}: i=1, \ldots, S\right\}$ be a partition of $\mathbb{R}^{p}$ into pairwise disjoint Borel sets, and let $\alpha_{i}, i=1, \ldots, S$, as well as $\beta_{i j}, i=1, \ldots, S, j=1, \ldots, q$, be real coefficients. Then we call a process $\left(X_{k}\right)_{k \in \mathbb{N}_{0}}$ satisfying

$$
X_{k}=\sum_{i=1}^{S}\left(\alpha_{i}+\sum_{j=1}^{q} \beta_{i j} X_{k-j}\right) \mathbf{1}_{\left\{\left(X_{k-d_{1}}, \ldots, X_{k-d_{p}}\right) \in J_{i}\right\}}+Z_{k}, \quad k \geq \max \left\{q, d_{p}\right\},
$$

\footnotetext{
Received 17 January 2008; revision received 19 February 2009.

* Postal address: Allianz Investment Management SE, Königinstrasse 28, 80802 München, Germany.

** Postal address: Center for Mathematical Sciences, Technische Universität München, Boltzmannstrasse 3, D-85747 Garching, Germany. Email address: fasen@ma.tum.de

Financial support from the Deutsche Forschungsgemeinschaft through a research grant is gratefully acknowledged.

*** Postal address: Institute for Mathematical Stochastics, Technische Universität Braunschweig, Pockelsstrasse 14, D-38106 Braunschweig, Germany. Email address: a.lindner@tu-bs.de
} 
and for which the starting vector $\left(X_{0}, \ldots, X_{\max \left\{q, d_{p}\right\}-1}\right)$ is independent of $\left(Z_{k+h}\right)_{h \in \mathbb{N}_{0}}$, a $\operatorname{TAR}(S, q)$ process. The current regime at time $k$ is determined by the vector $\left(X_{k-d_{1}}, \ldots\right.$, $\left.X_{k-d_{p}}\right)$ of the past observations, and within each regime, $\left(X_{k}\right)$ follows an $\operatorname{AR}\left(q_{i}\right)$ process with $q_{i}:=\max \left\{j \in\{1, \ldots, q\}: \beta_{i j} \neq 0\right\}$. Sometimes we also allow the variance of the noise to be regime dependent, by replacing $Z_{k}$ in (1.1) by $\sigma_{i} Z_{k}$, where $\sigma_{i}$ depends on the past in the same way as $\alpha_{i}$ does, but in this paper we will not consider such a specification. Autoregressive threshold models were introduced by Tong [28] in 1977 and were systematically presented by Tong and Lim [30], who used them as a model for the lynx data. Since then they have found various applications in many areas, such as financial economics, physics, population dynamics, and neural sciences, to name just a few; see the presentation in [13] for further information and references. In particular, when used as a model for financial data, it is important to have information about the tail and the extremal behaviors of these models, since stylized facts of financial data are heavy tails and clusters on high levels. The present paper will investigate the tail and the extremal behaviors of TAR models for various classes of driving noise sequences.

A somewhat related paper by Diop and Guegan [10] considered the tail behavior of TAR stochastic volatility models. Observe, however, that the threshold model under consideration in [10] is governed by a different regime switching mechanism, where the regime is not determined by the size of the previous observation, as for the TAR process, but by the sign of the volatility model.

The paper is organized as follows. In Section 2 we collect some known assumptions under which the model has a strictly stationary and geometrically ergodic solution. We then prove that under the given conditions the tail of the stationary solution can be estimated by the tail of a certain corresponding $\operatorname{AR}(q)$ sequence, a lemma which will turn out to be a crucial ingredient for the determination of the tail behavior.

Next, in Section 3 we derive the tail and the extremal behaviors of a TAR process with regularly varying noise. It is shown that the tail of the TAR model is O-regularly varying but not necessarily regularly varying. For this reason, we restrict our attention to the classical $\operatorname{TAR}(S, 1)$ model of order 1 with $S$ regimes, where the partition is a partition into $S$ intervals and the regime is determined by whether $X_{k-1}$ is in these intervals. In this case we show that the stationary solution has a regularly varying tail, and even that the finite-dimensional distributions are multivariate regularly varying. Furthermore, we derive the extremal behavior by point process convergence. A result is that the classical $\operatorname{TAR}(S, 1)$ process models extremal clusters.

Finally, Section 4 is also on general $\operatorname{TAR}(S, q)$ models with noise which has at most an exponentially decreasing tail without being regularly varying. It is then shown that the tail of the TAR model is in the same class and its extremal behavior is determined. Here, the sequence of point processes converges to a Poisson random measure, which reflects, in contrast to the regularly varying case, the absence of extremal clusters. Some of the results presented in this paper can also be found in the diploma thesis [3] of the first author.

Throughout the paper, we will denote $\mathbb{N}=\{1,2, \ldots\}, \mathbb{N}_{0}=\mathbb{N} \cup\{0\}, \mathbb{R}_{+}=(0, \infty)$, $\overline{\mathbb{R}}=\mathbb{R} \cup\{+\infty\} \cup\{-\infty\}$, and use $\stackrel{\text { W }}{\rightarrow}$ ' to denote weak convergence. For the integer part of a real number $x$, we write $\lfloor x\rfloor=\sup \{n \in \mathbb{Z}: n \leq x\}$. The Dirac measure at a point $x$ will be denoted by $\varepsilon_{x}$. For two strictly positive functions $g$ and $h$ and a constant $c \in[0, \infty)$, we write $g(t) \sim \operatorname{ch}(t)$ as $t \rightarrow \infty$ if the quotient $g(t) / h(t)$ tends to $c$ as $t \rightarrow \infty$. For $\boldsymbol{x} \in \mathbb{R}^{d}$, we denote by $\boldsymbol{x}^{\top}$ the transpose of $\boldsymbol{x}$ and by $\|\boldsymbol{x}\|$ the maximum norm of $\boldsymbol{x}$. As usual, the positive part and the negative part of $x \in \mathbb{R}$ is denoted by $x^{+}=\max \{x, 0\}$ and $x^{-}=\max \{0,-x\}$, respectively. The tail of a distribution function $F$ will be written as $\bar{F}=1-F$. 


\section{Model assumptions and basic properties}

In this paper we restrict our attention to noise sequences $\left(Z_{k}\right)$ which are subexponential (which includes regularly varying noise) or are in the class $\mathcal{L}(\gamma)$ with $\gamma>0$, which includes tails of the form $\mathrm{P}\left(Z_{1}>x\right) \sim K x^{b} \mathrm{e}^{-\gamma x}$ as $x \rightarrow \infty$ with $b \in \mathbb{R}$ and $K>0$. When determining the tail behavior, we will further assume the following classical tail balance condition, which is also standard for extreme value theory of linear ARMA processes, as presented, in, e.g. [12, Section A.3.3].

Condition 2.1. (Tail balance (TB).) There are constants $p^{+}, p^{-} \in[0,1]$ such that $p^{+}+p^{-}=1$, and the tail of $Z_{1}$ satisfies the TB condition if

$$
\mathrm{P}\left(Z_{1}>x\right) \sim p^{+} \mathrm{P}\left(\left|Z_{1}\right|>x\right) \text { and } \mathrm{P}\left(Z_{1}<-x\right) \sim p^{-} \mathrm{P}\left(\left|Z_{1}\right|>x\right) \text { as } x \rightarrow \infty .
$$

For the existence of strictly stationary solutions $\left(X_{k}\right)_{k \in \mathbb{N}_{0}}$, we use the following sufficient condition on the distribution of $Z_{1}$ and the size of the coefficients, which is sufficiently general for our purposes. That it is not a necessary condition for stationarity can be seen from the presentation in [6].

Condition 2.2. Let $\left(Z_{k}\right)_{k \in \mathbb{N}_{0}}$ be an i.i.d. sequence whose marginal distribution has a Lebesgue density $h$ satisfying $\inf _{x \in K} h(x)>0$ for every compact set $K \subset \mathbb{R}$. Furthermore, assume that $\mathrm{E}\left|Z_{1}\right|^{\min \{1, \eta\}}<\infty$ for some $\eta>0$. Denoting $\alpha:=\max _{k=1, \ldots, S}\left|\alpha_{k}\right|$ and $\beta_{j}:=$ $\max _{i=1, \ldots, S}\left|\beta_{i j}\right|$, assume further that $\beta:=\sum_{j=1}^{q} \beta_{j}<1$.

Define the function $f: \mathbb{R}^{l} \rightarrow \mathbb{R}$ by

$$
f\left(x_{k-1}, x_{k-2}, \ldots, x_{k-l}\right):=\sum_{i=1}^{S}\left(\alpha_{i}+\sum_{j=1}^{q_{i}} \beta_{i j} x_{k-j}\right) \mathbf{1}_{\left\{\left(x_{k-d_{1}}, \ldots, x_{k-d_{p}}\right) \in J_{i}\right\}},
$$

where $l:=\max \left\{q, d_{p}\right\}$, and define $\boldsymbol{X}_{k}:=\left(X_{k}, X_{k-1}, \ldots, X_{k-l+1}\right)^{\top}$. Then the threshold model (1.1) has the representation

$$
X_{k}=f\left(X_{k-1}\right)+Z_{k} \quad \text { for } k \in\{l, l+1, \ldots\},
$$

with $f\left(\boldsymbol{X}_{k-1}\right)$ being independent of $Z_{k}$. Furthermore, we write $\boldsymbol{Z}_{k}:=\left(Z_{k}, 0, \ldots, 0\right)^{\top}$ and $\boldsymbol{f}(\boldsymbol{x}):=\left(f(\boldsymbol{x}), x_{1}, \ldots, x_{l-1}\right)^{\top}$ for $\boldsymbol{x}:=\left(x_{1}, \ldots, x_{l}\right)^{\top} \in \mathbb{R}^{l}$. Then $\left(\boldsymbol{X}_{k}\right)_{k \geq l-1}$ is a Markov chain, where

$$
\boldsymbol{X}_{k}=\boldsymbol{f}\left(\boldsymbol{X}_{k-1}\right)+\boldsymbol{Z}_{k} \quad \text { for } k \geq l .
$$

The following lemma assures the existence of a geometrically ergodic, strictly stationary solution. Geometric ergodicity has already been proved in [5] (cf. [29, Example A1.2, p. 464]) and [1, Theorem 3.2 and Example 3.6], under the slightly more restrictive condition that $\mathrm{E}\left|Z_{1}\right|<\infty$. However, the proof in [1] can easily be generalized to the case where only finiteness of $\mathrm{E}\left|Z_{1}\right|^{\min \{1, \eta\}}$ for some $\eta>0$ is assumed: simply replace the test function $\left(x_{1}, \ldots, x_{p}\right) \mapsto \max \left\{\left|x_{1}\right|, \ldots,\left|x_{p}\right|\right\}$ appearing in the proof of Theorem 3.2 of [1] by $\left(x_{1}, \ldots, x_{p}\right) \mapsto\left(\max \left\{\left|x_{1}\right|, \ldots,\left|x_{p}\right|\right\}\right)^{\min \{1, \eta\}}$. Also, note that the proof in [1] carries over to the more general partitions considered in (1.1) without change. That geometric ergodicity of the stationary solution then implies strong mixing with geometrically decreasing mixing rate in the sense that

$$
\sup _{A \in \sigma\left(X_{j}: j \leq m\right), B \in \sigma\left(X_{j}: j \geq k+m\right)}|\mathrm{P}(A \cap B)-\mathrm{P}(A) \mathrm{P}(B)| \leq K \gamma^{k}, \quad k, m \in \mathbb{N},
$$

for some $0<\gamma<1$ and $K>0$, then follows from [21, Theorem 16.1.5]. 
Lemma 2.1. Let $\left(X_{k}\right)_{k \in \mathbb{N}_{0}}$ be a TAR process as given in (1.1), and suppose that Condition 2.2 holds. Then $\left(X_{k}\right)_{k \in \mathbb{N}_{0}}$ is geometrically ergodic and admits a unique strictly stationary solution, which is strongly mixing with geometrically decreasing mixing rate in the sense of (2.1).

The next lemma is crucial for the analysis of extremes of TAR models. It states that, under our assumptions, there is a causal $\operatorname{AR}(q)$ process whose stationary solution has a tail which is not smaller than that of the stationary solution of the TAR process.

Lemma 2.2. Suppose that Condition 2.2 holds, and let $\left(X_{k}\right)_{k \in \mathbb{N}_{0}}$ be the stationary solution of the TAR model (1.1). Let $\left(\tilde{Z}_{k}\right)_{k \in \mathbb{Z}}$ be an i.i.d. sequence such that $\tilde{Z}_{k}=\left|Z_{k}\right|+\alpha$ for $k \in \mathbb{N}_{0}$. Denote by $\left(\tilde{X}_{k}\right)_{k \in \mathbb{Z}}$ the unique strictly stationary solution of the causal $A R(q)$ process

$$
\tilde{X}_{k}:=\sum_{j=1}^{q} \beta_{j} \tilde{X}_{k-j}+\tilde{Z}_{k}, \quad k \in \mathbb{Z} .
$$

Then $\left(\tilde{X}_{k}\right)_{k \in \mathbb{Z}}$ has the almost-surely convergent moving average representation

$$
\tilde{X}_{k}=\sum_{j=0}^{\infty} \psi_{j} \tilde{Z}_{k-j}
$$

where $\psi_{0}=1,0 \leq \psi_{j}<1$ for $j \in \mathbb{N}$, and $\left(\psi_{j}\right)_{j \in \mathbb{N}_{0}}$ is bounded by a geometrically decreasing sequence, i.e. $\psi_{j} \leq K \gamma^{j}$ for some $0<\gamma<1$ and $K>0$. Furthermore, for any $m \in \mathbb{N}$, $k_{1}, \ldots, k_{m} \in \mathbb{N}_{0}$, and $x_{1}, \ldots, x_{m} \in \mathbb{R}$, it holds that

$$
\mathrm{P}\left(\left|X_{k_{1}}\right|>x_{1}, \ldots,\left|X_{k_{m}}\right|>x_{m}\right) \leq \mathrm{P}\left(\tilde{X}_{k_{1}}>x_{1}, \ldots, \tilde{X}_{k_{m}}>x_{m}\right) .
$$

Proof. Since the polynomial $\Phi(z):=1-\sum_{j=1}^{q} \beta_{j} z^{j}$ has no zeros for $|z| \leq 1$ as a consequence of $\sum_{j=1}^{q} \beta_{j}<1$, it follows that process (2.2) is causal. Expanding $\Phi(z)^{-1}=$ $\sum_{j=0}^{\infty} \psi_{j} z^{j}$ in a power series around the origin gives $\psi_{0}=1, \psi_{1}=\beta_{1}$, and the recursions $\psi_{m}=\sum_{j=\max \{0, m-q\}}^{m-1} \beta_{m-j} \psi_{j}$ for $m \geq 2$. A simple induction argument then shows that $0 \leq \psi_{j}<1$ for $j \geq 1$ and that $\left(\psi_{m}\right)$ can be dominated by an exponentially decreasing sequence. In particular, $\sum_{j=0}^{\infty} \psi_{j}^{\min \{1, \eta\}}<\infty$. Since $\mathrm{E}\left|\tilde{Z}_{1}\right|^{\min \{1, \eta\}}<\infty$ by assumption, this gives almost-sure convergence of (2.3).

Define the sequence $\left(X_{k}^{*}\right)_{k \in \mathbb{N}_{0}}$ by $X_{0}^{*}:=\left|X_{0}\right|, \ldots, X_{q-1}^{*}:=\left|X_{q-1}\right|$ and

$$
X_{k}^{*}:=\sum_{j=1}^{q} \beta_{j} X_{k-j}^{*}+\tilde{Z}_{k}, \quad k \geq q .
$$

Then it follows by induction that

$$
\left|X_{k}\right| \leq X_{k}^{*} \quad \text { for all } k \in \mathbb{N}_{0},
$$

since

$$
\left|X_{k}\right| \leq \max _{i=1, \ldots, S} \alpha_{i}+\sum_{j=1}^{q} \max _{i=1, \ldots, S}\left|\beta_{i j}\right|\left|X_{k-j}\right|+\left|Z_{k}\right| \leq \sum_{j=1}^{q} \beta_{j} X_{k-j}^{*}+\tilde{Z}_{k}=X_{k}^{*} .
$$

Observe that $\left(X_{t_{1}+n}^{*}, \ldots, X_{t_{m}+n}^{*}\right)$ converges in distribution as $n \rightarrow \infty$ to the stationary solution $\left(\tilde{X}_{t_{1}}, \ldots, \tilde{X}_{t_{m}}\right)$ of the causal AR $(q)$ process $(2.2)$, and that the topological boundary of the set 
$\left[x_{1}, \infty\right) \times \cdots \times\left[x_{m}, \infty\right)$ has $\mathrm{P}_{\left(\tilde{X}_{t_{1}}, \ldots, \tilde{X}_{t_{m}}\right)}$-measure 0 as a consequence of the absolute continuity of the distribution of $Z_{1}$. Thus, we conclude from (2.5) that

$$
\begin{aligned}
\mathrm{P}\left(X_{t_{1}}>x_{1}, \ldots, X_{t_{m}}>x_{m}\right) & =\lim _{n \rightarrow \infty} \mathrm{P}\left(X_{t_{1}+n}>x_{1}, \ldots, X_{t_{m}+n}>x_{m}\right) \\
& \leq \limsup _{n \rightarrow \infty} \mathrm{P}\left(X_{t_{1}+n}^{*}>x_{1}, \ldots, X_{t_{m}+n}^{*}>x_{m}\right) \\
& =\mathrm{P}\left(\tilde{X}_{t_{1}}>x_{1}, \ldots, \tilde{X}_{t_{m}}>x_{m}\right)
\end{aligned}
$$

showing (2.4).

\section{Regularly varying noise}

Recall that a measurable function $f:(0, \infty) \rightarrow(0, \infty)$ is said to be regularly varying (at $\infty)$ with index $-\kappa \in \mathbb{R}$, written as $f \in \mathcal{R}_{-\kappa}$, if

$$
\lim _{x \rightarrow \infty} \frac{f(x u)}{f(x)}=u^{-\kappa} \quad \text { for all } u>0 .
$$

Functions in $\mathcal{R}_{0}$ are also called slowly varying functions and, for $\kappa \geq 0$, it holds that $f \in \mathcal{R}_{-\kappa}$ if and only if $f(x)=x^{-\kappa} L(x)$ for all $x>0$ with a slowly varying function $L$. For a random variable $Z$ with distribution function $F_{Z}$, we also write $Z \in \mathcal{R}_{-\kappa}$ to indicate that $Z$ has a regularly varying tail, i.e. that $\bar{F}_{Z} \in \mathcal{R}_{-\kappa}$. Examples of distributions having regularly varying tails include Pareto distributions and $\alpha$-stable distributions with $\alpha \in(0,2)$.

\subsection{O-regular variation of TAR models}

Unlike for linear models such as ARMA processes, stationary solutions of general TAR models with regularly varying noise give only O-regularly varying tails. Recall that a measurable function $f:(0, \infty) \rightarrow(0, \infty)$ is called $O$-regularly varying $($ at $\infty)$ if

$$
0<\liminf _{x \rightarrow \infty} \frac{f(x u)}{f(x)} \leq \limsup _{x \rightarrow \infty} \frac{f(x u)}{f(x)}<\infty \quad \text { for all } u>0 .
$$

Clearly, every regularly varying function is O-regularly varying. For TAR processes with regularly varying noise, we now have the following result.

Lemma 3.1. (O-regular variation.) Suppose that Conditions 2.1 and 2.2 hold, and let $\left(X_{k}\right)_{k \in \mathbb{N}_{0}}$ be a stationary version of the TAR process as given in (1.1). Suppose further that $\left|Z_{1}\right| \in \mathcal{R}_{-\kappa}$ for some $\kappa>0$. Then

$$
p^{+} 2^{-\kappa} \leq \liminf _{x \rightarrow \infty} \frac{\mathrm{P}\left(X_{0}>x\right)}{\mathrm{P}\left(\left|Z_{1}\right|>x\right)} \leq \limsup _{x \rightarrow \infty} \frac{\mathrm{P}\left(X_{0}>x\right)}{\mathrm{P}\left(\left|Z_{1}\right|>x\right)} \leq \sum_{j=0}^{\infty} \psi_{j}^{\kappa},
$$

where $\left(\psi_{j}\right)_{j \in \mathbb{N}_{0}}$ is given as in Lemma 2.2. In particular, $\bar{F}_{X}$ is $O$-regularly varying if $p^{+}>0$.

Proof. From Lemma 2.2 and [24, Lemma 4.24], we obtain

$$
\limsup _{x \rightarrow \infty} \frac{\mathrm{P}\left(X_{0}>x\right)}{\mathrm{P}\left(\left|Z_{1}\right|>x\right)} \leq \limsup _{x \rightarrow \infty} \frac{\mathrm{P}\left(\tilde{X}_{0}>x\right)}{\mathrm{P}\left(\left|Z_{1}\right|>x\right)}=\sum_{j=0}^{\infty} \psi_{j}^{\kappa}
$$


On the other hand, since $X_{k}-Z_{k}$ is independent of $Z_{k}$, it also holds that

$$
\liminf _{x \rightarrow \infty} \frac{\mathrm{P}\left(X_{0}>x\right)}{\mathrm{P}\left(\left|Z_{1}\right|>x\right)} \geq \liminf _{x \rightarrow \infty} \frac{\mathrm{P}\left(Z_{1}>2 x\right)}{\mathrm{P}\left(\left|Z_{1}\right|>x\right)} \mathrm{P}\left(X_{1}-Z_{1}>-x\right)=p^{+} 2^{-\kappa} .
$$

This gives (3.2), implying O-regular variation of the tail of $X_{0}$ if $p^{+}>0$.

The next proposition shows that without specific assumptions on the partition, regular variation of the stationary distribution cannot be expected, even for a $\operatorname{TAR}(2,1)$ model.

Proposition 3.1. Let $\left(Z_{k}\right)_{k \in \mathbb{N}_{0}}$ be an i.i.d. sequence such that $\mathrm{P}\left(Z_{1}>x\right) \sim x^{-\kappa}$ as $x \rightarrow \infty$ for some $\kappa>0$ and that Conditions 2.1 and 2.2 hold with $p^{+}>0$. For the partition $J_{1}:=$ $\bigcup_{m \in \mathbb{N}_{0}}\left(4^{m}, 4^{m+1 / 2}\right]$ and $J_{2}:=\mathbb{R} \backslash J_{1}$, consider the TAR $(2,1)$ model

$$
X_{k}=\left\{\begin{array}{ll}
\beta_{1} X_{k-1}+Z_{k} & \text { for } X_{k-1} \in J_{1}, \\
Z_{k} & \text { for } X_{k-1} \in J_{2},
\end{array} \quad k \in \mathbb{N},\right.
$$

where $0<\beta_{1}<1$. Then there are constants $0<c_{1}<c_{2}<\infty$ such that the stationary solution $\left(X_{k}\right)_{k \in \mathbb{N}_{0}}$ of the TAR $(2,1)$ model with distribution function $F_{X}$ satisfies

$$
c_{1} x^{-\kappa} \leq \bar{F}_{X}(x) \leq c_{2} x^{-\kappa}, \quad x \geq 1,
$$

but $\bar{F}_{X}$ is not regularly varying.

Proof. Equation (3.3) follows immediately from Lemma 3.1. Let $L$ be the function satisfying

$$
\bar{F}_{X}(x)=\mathrm{P}\left(X_{0}>x\right)=L(x) x^{-\kappa}, \quad x>0 .
$$

By (3.3), it follows that $c_{1} \leq L(x) \leq c_{2}$ for $x \geq 1$. For $k \in \mathbb{N}_{0}$, define

$$
W_{k}:= \begin{cases}\beta_{1} X_{k} & \text { for } X_{k} \in J_{1} \\ 0 & \text { for } X_{k} \in J_{2}\end{cases}
$$

We will show that the assumption that $\bar{F}_{X}$ is regularly varying gives an O-regularly varying tail of $W_{k-1}$ which is not regularly varying, and then obtain a contradiction of the tail behavior of $X_{k}=W_{k-1}+Z_{k}$, where $W_{k-1}$ and $Z_{k}$ are independent.

So we assume that $\bar{F}_{X}$ is regularly varying. Then it follows that $L$ must be slowly varying. We will first show that there are constants $0<d_{1} \leq d_{2}<\infty$ such that

$$
d_{1} x^{-\kappa} \leq \mathrm{P}\left(W_{k}>x\right) \leq d_{2} x^{-\kappa}, \quad x \geq 1 .
$$

Here, the right-hand side inequality follows easily from $\mathrm{P}\left(W_{k}>x\right) \leq \mathrm{P}\left(\beta_{1} X_{k}>x\right)$ and (3.3). For the left-hand side inequality, observe that the regular variation of $\bar{F}_{X}$ implies that

$$
\begin{aligned}
\lim _{x \rightarrow \infty} \frac{\mathrm{P}\left(X_{k} \in(x, 2 x]\right)}{\mathrm{P}\left(X_{k} \in(x, 4 x]\right)} & =\lim _{x \rightarrow \infty} \frac{\mathrm{P}\left(X_{k}>x\right)-\mathrm{P}\left(X_{k}>2 x\right)}{\mathrm{P}\left(X_{k}>x\right)-\mathrm{P}\left(X_{k}>4 x\right)} \\
& =\lim _{x \rightarrow \infty} \frac{\left[\mathrm{P}\left(X_{k}>x\right)-\mathrm{P}\left(X_{k}>2 x\right)\right] / \mathrm{P}\left(X_{k}>x\right)}{\left[\mathrm{P}\left(X_{k}>x\right)-\mathrm{P}\left(X_{k}>4 x\right)\right] / \mathrm{P}\left(X_{k}>x\right)} \\
& =\frac{1-2^{-\kappa}}{1-4^{-\kappa}} .
\end{aligned}
$$


For $x>0$, denote by $m(x)$ the unique nonnegative integer such that $4^{m(x)-1}<x / \beta_{1} \leq 4^{m(x)}$. Then, given $\varepsilon>0$, it follows that, for large enough $x \geq x(\varepsilon)$,

$$
\begin{aligned}
\mathrm{P}\left(W_{k}>x\right) & =\sum_{m=0}^{\infty} \mathrm{P}\left(X_{k}>\frac{x}{\beta_{1}}, X_{k} \in\left(4^{m}, 4^{m+1 / 2}\right]\right) \\
& \geq \sum_{m=m(x)}^{\infty} \mathrm{P}\left(X_{k} \in\left(4^{m}, 4^{m+1 / 2}\right]\right) \\
& \geq(1-\varepsilon) \frac{1-2^{-\kappa}}{1-4^{-\kappa}} \sum_{m=m(x)}^{\infty} \mathrm{P}\left(X_{k} \in\left(4^{m}, 4^{m+1}\right]\right) \\
& =(1-\varepsilon) \frac{1-2^{-\kappa}}{1-4^{-\kappa}} \mathrm{P}\left(X_{k}>4^{m(x)}\right) .
\end{aligned}
$$

The left-hand side inequality in (3.4) then follows from the corresponding one in (3.3) and $4^{m(x)}<4 x / \beta_{1}$. Thus, it follows from (3.4) that we can write

$$
\mathrm{P}\left(W_{k}>x\right)=r(x) x^{-\kappa}, \quad x \in \mathbb{R},
$$

where $d_{1} \leq r(x) \leq d_{2}$ for $x \geq 1$. Now, let $\left(x_{m}\right)_{m \in \mathbb{N}}$ be a sequence of numbers such that $x_{m} / \beta_{1} \in\left[4^{m+4 / 6}, 4^{m+5 / 6}\right]$. Then $\lambda x_{m} / \beta_{1} \in\left(4^{m+1 / 2}, 4^{m+1}\right)$ for every $\lambda \in\left(4^{-1 / 6}, 4^{1 / 6}\right)$, so that

$$
\mathrm{P}\left(W_{k}>\lambda x_{m}\right)=\mathrm{P}\left(X_{k}>\frac{\lambda x_{m}}{\beta_{1}}, X_{k} \in J_{1}\right)=\mathrm{P}\left(X_{k}>\frac{x_{m}}{\beta_{1}}, X_{k} \in J_{1}\right)=\mathrm{P}\left(W_{k}>x_{m}\right),
$$

giving

$$
r\left(\lambda x_{m}\right)=\lambda^{\kappa} r\left(x_{m}\right) \quad \text { for all } \lambda \in\left(4^{-1 / 6}, 4^{1 / 6}\right) \text { and } m \in \mathbb{N} .
$$

This implies in particular that $r$ is not slowly varying, so that the distribution function of $W_{k}$ cannot have a regularly varying tail by (3.5).

Choose $\delta>0$ such that $4^{-1 / 6}<1-\delta<1+\delta<4^{1 / 6}$, and let $x^{\prime}:=(1+\delta) x$ and $x^{\prime \prime}:=(1-\delta) x$. Write $\mathrm{P}\left(Z_{1}>x\right)=q(x) x^{-\kappa}$, so that $\lim _{x \rightarrow \infty} q(x)=1$. Then, using exactly the same proof as in [17, p. 278], it follows that, for given $\varepsilon>0$ and large enough $x \geq x(\varepsilon)$, the tail of $X_{k}=W_{k-1}+Z_{k}$ satisfies

$$
(1-\varepsilon)\left(r\left(x^{\prime}\right)+q\left(x^{\prime}\right)\right)\left(x^{\prime}\right)^{-\kappa} \leq \mathrm{P}\left(X_{k}>x\right) \leq(1+\varepsilon)\left(r\left(x^{\prime \prime}\right)+q\left(x^{\prime \prime}\right)\right)\left(x^{\prime \prime}\right)^{-\kappa} .
$$

Choosing $x_{m}$ as before, the left-hand side inequality of (3.7) together with (3.6) shows that, for large enough $m$,

$$
\begin{aligned}
& \frac{\mathrm{P}\left(X_{k}>x_{m}\right)}{\left(r\left(x_{m}\right)+q\left(x_{m}\right)\right) x_{m}^{-\kappa}} \\
& \quad \geq(1-\varepsilon)(1+\delta)^{-\kappa} \frac{r\left(x_{m}\right)(1+\delta)^{\kappa}+q\left(x_{m}^{\prime}\right)}{r\left(x_{m}\right)+q\left(x_{m}\right)} \\
& \quad=(1-\varepsilon)(1+\delta)^{-\kappa}\left(1+\frac{r\left(x_{m}\right)\left((1+\delta)^{\kappa}-1\right)+q\left(x_{m}^{\prime}\right)-q\left(x_{m}\right)}{r\left(x_{m}\right)+q\left(x_{m}\right)}\right) .
\end{aligned}
$$


Using $d_{1} \leq r(x) \leq d_{2}$ for $x \geq 1, \lim _{x \rightarrow \infty} q(x)=1$, and $\lim _{\delta \rightarrow 0}(1+\delta)^{\kappa}-1=0$, we conclude that

$$
\liminf _{m \rightarrow \infty} \frac{\mathrm{P}\left(X_{k}>x_{m}\right)}{\left(r\left(x_{m}\right)+q\left(x_{m}\right)\right) x_{m}^{-\kappa}} \geq 1 .
$$

A similar argument holds for the limes superior, so that

$$
L\left(x_{m}\right) \sim\left(r\left(x_{m}\right)+q\left(x_{m}\right)\right) \quad \text { as } m \rightarrow \infty .
$$

Taking for $x_{m}$ the sequences $u_{m}:=4^{m+9 / 12} \beta_{1}$ and $v_{m}:=4^{m+10 / 12} \beta_{1}=4^{1 / 12} u_{m}$, it follows, from (3.6) and (3.8), that

$$
\frac{L\left(v_{m}\right)}{L\left(u_{m}\right)} \sim \frac{r\left(v_{m}\right)+q\left(v_{m}\right)}{r\left(u_{m}\right)+q\left(u_{m}\right)} \sim \frac{4^{\kappa / 12} r\left(u_{m}\right)+q\left(u_{m}\right)}{r\left(u_{m}\right)+q\left(u_{m}\right)}=1+\frac{\left(4^{\kappa / 12}-1\right) r\left(u_{m}\right)}{r\left(u_{m}\right)+q\left(u_{m}\right)} \quad \text { as } m \rightarrow \infty,
$$

and the latter does not converge to 1 as $m \rightarrow \infty$, since $d_{1} \leq r\left(u_{m}\right) \leq d_{2}$ and $\lim _{x \rightarrow \infty} q(x)=1$. Hence, $L$ cannot be slowly varying, contradicting the regular variation of $\bar{F}_{X}$.

\subsection{Regular variation of $\operatorname{TAR}(S, 1)$ models with specific partitions}

In this and the next subsection we restrict our attention to stationary $\operatorname{TAR}(S, 1)$ models with representation

$$
X_{k}=\sum_{i=1}^{S}\left(\alpha_{i}+\beta_{i} X_{k-1}\right) \mathbf{1}_{\left\{X_{k-1} \in J_{i}\right\}}+Z_{k} \quad \text { for } k \in \mathbb{N}
$$

where $J_{1}=\left(-\infty, r_{1}\right], J_{2}=\left(r_{2}, \infty\right)$ for some $r_{1}, r_{2} \in \mathbb{R}, r_{1} \leq r_{2}$, and $\left\{J_{i}: i=3, \ldots, S\right\}$ is a measurable partition of $\left(r_{1}, r_{2}\right]$. For this model, we are able to compute the tail behavior explicitly, as we will show in the next lemma.

Lemma 3.2. (Regular variation.) Suppose that Conditions 2.1 and 2.2 hold, and let $\left(X_{k}\right)_{k \in \mathbb{N}_{0}}$ be a stationary version of the TAR(S,1) process as given in (3.9). Suppose further that $\left|Z_{1}\right| \in$ $\mathcal{R}_{-\kappa}$ for some $\kappa>0$. Then $\left|X_{0}\right| \in \mathcal{R}_{-\kappa}$. More precisely, defining

$$
\beta_{i}^{+}:=\left\{\begin{array}{ll}
\beta_{i}, & \beta_{i}>0, \\
0, & \beta_{i} \leq 0,
\end{array} \quad \text { and } \beta_{i}^{-}:= \begin{cases}\left|\beta_{i}\right|, & \beta_{i}<0 \\
0, & \beta_{i} \geq 0\end{cases}\right.
$$

for $i=1,2$, it holds that

$$
\lim _{x \rightarrow \infty} \frac{\mathrm{P}\left(X_{0}>x\right)}{\mathrm{P}\left(\left|Z_{1}\right|>x\right)}=\frac{p^{+}+p^{-}\left(\beta_{1}^{-}\right)^{\kappa}}{1-\left(\beta_{2}^{+}\right)^{\kappa}-\left(\beta_{1}^{-}\right)^{\kappa}\left(\beta_{2}^{-}\right)^{\kappa}}=: \tilde{p}^{+}
$$

and

$$
\lim _{x \rightarrow \infty} \frac{\mathrm{P}\left(X_{0}<-x\right)}{\mathrm{P}\left(\left|Z_{1}\right|>x\right)}=\frac{p^{-}+p^{+}\left(\beta_{2}^{-}\right)^{\kappa}}{1-\left(\beta_{1}^{+}\right)^{\kappa}-\left(\beta_{2}^{-}\right)^{\kappa}\left(\beta_{1}^{-}\right)^{\kappa}}=: \tilde{p}^{-} .
$$

In particular, $X_{0} \in \mathcal{R}_{-\kappa}$ if $\tilde{p}^{+}>0$. Furthermore, $\tilde{p}^{+} \geq p^{+}$and $\tilde{p}^{-} \geq p^{-}$. 
Proof. Let $x>0$ be fixed, and let $\left(a_{n}\right)_{n \in \mathbb{N}}$ be a sequence of positive real numbers tending to $\infty$ as $n \rightarrow \infty$. Then, for any $\delta>0$, we can write

$$
\begin{aligned}
\mathrm{P}\left(X_{1}>x a_{n}\right)= & \mathrm{P}\left(X_{1}>x a_{n},\left|Z_{1}\right|>\delta a_{n},\left|X_{0}\right|>\delta a_{n}\right) \\
& +\mathrm{P}\left(X_{1}>x a_{n},\left|Z_{1}\right|>\delta a_{n},\left|X_{0}\right| \leq \delta a_{n}\right) \\
& +\mathrm{P}\left(X_{1}>x a_{n},\left|Z_{1}\right| \leq \delta a_{n},\left|X_{0}\right| \leq \delta a_{n}\right) \\
& +\mathrm{P}\left(X_{1}>x a_{n},\left|Z_{1}\right| \leq \delta a_{n}, X_{0}>\delta a_{n}\right) \\
& +\mathrm{P}\left(X_{1}>x a_{n},\left|Z_{1}\right| \leq \delta a_{n}, X_{0}<-\delta a_{n}\right) \\
= & I+I I+I I I+I V+V, \text { say. }
\end{aligned}
$$

We will study the tail behavior of the five summands of (3.12). Using the independence of $Z_{1}$ and $X_{0}$, we obtain for the first term

$$
\lim _{n \rightarrow \infty} \frac{I}{\mathrm{P}\left(\left|Z_{1}\right|>x a_{n}\right)} \leq \lim _{n \rightarrow \infty} \frac{\mathrm{P}\left(\left|Z_{1}\right|>\delta a_{n}\right) \mathrm{P}\left(\left|X_{0}\right|>\delta a_{n}\right)}{\mathrm{P}\left(\left|Z_{1}\right|>x a_{n}\right)}=0 .
$$

For the second term of (3.12), observe that

$$
\begin{aligned}
\limsup _{n \rightarrow \infty} \frac{\mathrm{P}\left(X_{1}>x a_{n},\left|Z_{1}\right|>\delta a_{n},\left|X_{0}\right| \leq \delta a_{n}\right)}{\mathrm{P}\left(\left|Z_{1}\right|>x a_{n}\right)} & \leq \limsup _{n \rightarrow \infty} \frac{\mathrm{P}\left(Z_{1}>(x-\beta \delta) a_{n}-\alpha\right)}{\mathrm{P}\left(\left|Z_{1}\right|>x a_{n}\right)} \\
& =p^{+} \frac{(x-\beta \delta)^{-\kappa}}{x^{-\kappa}} .
\end{aligned}
$$

On the other hand,

$$
\begin{aligned}
\liminf _{n \rightarrow \infty} & \frac{\mathrm{P}\left(X_{1}>x a_{n},\left|Z_{1}\right|>\delta a_{n},\left|X_{0}\right| \leq \delta a_{n}\right)}{\mathrm{P}\left(\left|Z_{1}\right|>x a_{n}\right)} \\
\geq & \liminf _{n \rightarrow \infty} \frac{\mathrm{P}\left(Z_{1}>(x+\beta \delta) a_{n}+\alpha\right)-\mathrm{P}\left(Z_{1}>(x+\beta \delta) a_{n}+\alpha,\left|X_{0}\right|>\delta a_{n}\right)}{\mathrm{P}\left(\left|Z_{1}\right|>x a_{n}\right)} \\
& =p^{+} \frac{(x+\beta \delta)^{-\kappa}}{x^{-\kappa}} \text { for } 0<\delta<x,
\end{aligned}
$$

and we conclude that

$$
\lim _{\delta \downarrow 0} \liminf _{n \rightarrow \infty} \frac{I I}{\mathrm{P}\left(\left|Z_{1}\right|>x a_{n}\right)}=\lim _{\delta \downarrow 0} \limsup _{n \rightarrow \infty} \frac{I I}{\mathrm{P}\left(\left|Z_{1}\right|>x a_{n}\right)}=p^{+} .
$$

The third term of (3.12) is 0 provided $\delta<x / 2$. For the investigation of $I V$ and $V$, we define

$$
\begin{array}{ll}
\bar{A}=\limsup _{u \rightarrow \infty} \frac{\mathrm{P}\left(X_{0}>u\right)}{\mathrm{P}\left(\left|Z_{1}\right|>u\right)}, & \underline{A}=\liminf _{u \rightarrow \infty} \frac{\mathrm{P}\left(X_{0}>u\right)}{\mathrm{P}\left(\left|Z_{1}\right|>u\right)}, \\
\bar{B}=\limsup _{u \rightarrow \infty} \frac{\mathrm{P}\left(X_{0}<-u\right)}{\mathrm{P}\left(\left|Z_{1}\right|>u\right)}, & \underline{B}=\liminf _{u \rightarrow \infty} \frac{\mathrm{P}\left(X_{0}<-u\right)}{\mathrm{P}\left(\left|Z_{1}\right|>u\right)} .
\end{array}
$$

All these terms are finite by Lemma 3.1. Then we obtain

$$
\lim _{\delta \downarrow 0} \limsup _{n \rightarrow \infty} \frac{I V}{\mathrm{P}\left(\left|Z_{1}\right|>x a_{n}\right)} \leq \lim _{\delta \downarrow 0} \limsup _{n \rightarrow \infty} \frac{\mathrm{P}\left(\alpha_{2}+\beta_{2}^{+} X_{0}>(x-\delta) a_{n}\right)}{\mathrm{P}\left(\left|Z_{1}\right|>x a_{n}\right)}=\bar{A}\left(\beta_{2}^{+}\right)^{\kappa},
$$


and, similarly,

$$
\begin{aligned}
\lim _{\delta \downarrow 0} \liminf _{n \rightarrow \infty} \frac{I V}{\mathrm{P}\left(\left|Z_{1}\right|>x a_{n}\right)} & \geq \lim _{\delta \downarrow 0} \liminf _{n \rightarrow \infty} \frac{\mathrm{P}\left(\alpha_{2}+\beta_{2}^{+} X_{0}>(x+\delta) a_{n}\right)}{\mathrm{P}\left(\left|Z_{1}\right|>x a_{n}\right)} \mathrm{P}\left(\left|Z_{1}\right| \leq \delta a_{n}\right) \\
& =\underline{A}\left(\beta_{2}^{+}\right)^{\kappa} .
\end{aligned}
$$

The bounds of $V$ are

$$
\lim _{\delta \downarrow 0} \limsup _{n \rightarrow \infty} \frac{V}{\mathrm{P}\left(\left|Z_{1}\right|>x a_{n}\right)} \leq \lim _{\delta \downarrow 0} \limsup _{n \rightarrow \infty} \frac{\mathrm{P}\left(\alpha_{1}-\beta_{1}^{-} X_{0}>(x-\delta) a_{n}\right)}{\mathrm{P}\left(\left|Z_{1}\right|>x a_{n}\right)}=\bar{B}\left(\beta_{1}^{-}\right)^{\kappa}
$$

and

$$
\begin{aligned}
\lim _{\delta \downarrow 0} \liminf _{n \rightarrow \infty} \frac{V}{\mathrm{P}\left(\left|Z_{1}\right|>x a_{n}\right)} & \geq \lim _{\delta \downarrow 0} \liminf _{n \rightarrow \infty} \frac{\mathrm{P}\left(\alpha_{1}-\beta_{1}^{-} X_{0}>(x+\delta) a_{n}\right)}{\mathrm{P}\left(\left|Z_{1}\right|>x a_{n}\right)} \mathrm{P}\left(\left|Z_{1}\right| \leq \delta a_{n}\right) \\
& =\underline{B}\left(\beta_{1}^{-}\right)^{\kappa} .
\end{aligned}
$$

Then (3.12)-(3.18) give

$$
p^{+}+\underline{A}\left(\beta_{2}^{+}\right)^{\kappa}+\underline{B}\left(\beta_{1}^{-}\right)^{\kappa} \leq \underline{A} \leq \bar{A} \leq p^{+}+\bar{A}\left(\beta_{2}^{+}\right)^{\kappa}+\bar{B}\left(\beta_{1}^{-}\right)^{\kappa} .
$$

Since

$$
-X_{k}=\sum_{i=1}^{S}\left(-\alpha_{i}\right)+\beta_{i}\left(-X_{k-1}\right) \mathbf{1}_{\left\{-X_{k-1} \in-J_{i}\right\}}-Z_{k},
$$

we obtain, by symmetry,

$$
p^{-}+\underline{B}\left(\beta_{1}^{+}\right)^{\kappa}+\underline{A}\left(\beta_{2}^{-}\right)^{\kappa} \leq \underline{B} \leq \bar{B} \leq p^{-}+\bar{B}\left(\beta_{1}^{+}\right)^{\kappa}+\bar{A}\left(\beta_{2}^{-}\right)^{\kappa} .
$$

If $\beta_{1} \geq 0$ then (3.19) gives

$$
\underline{A}=\bar{A}=\frac{p^{+}}{1-\left(\beta_{2}^{+}\right)^{\kappa}} .
$$

In the case in which $\beta_{1}<0$ we obtain, by (3.20),

$$
p^{-}+\underline{A}\left(\beta_{2}^{-}\right)^{\kappa} \leq \underline{B} \quad \text { and } \quad \bar{B} \leq p^{-}+\bar{A}\left(\beta_{2}^{-}\right)^{\kappa} .
$$

Inserting (3.21) into (3.19) yields

$$
\frac{p^{+}+p^{-}\left(\beta_{1}^{-}\right)^{\kappa}}{1-\left(\beta_{2}^{+}\right)^{\kappa}-\left(\beta_{2}^{-}\right)^{\kappa}\left(\beta_{1}^{-}\right)^{\kappa}} \leq \underline{A} \leq \bar{A} \leq \frac{p^{+}+p^{-}\left(\beta_{1}^{-}\right)^{\kappa}}{1-\left(\beta_{2}^{+}\right)^{\kappa}-\left(\beta_{2}^{-}\right)^{\kappa}\left(\beta_{1}^{-}\right)^{\kappa}},
$$

which gives the result $\underline{A}=\bar{A}=\tilde{p}^{+}$. Inserting this into (3.20) gives $\underline{B}=\bar{B}=\tilde{p}^{-}$also. That $\tilde{p}^{+} \geq p^{+}$and $\tilde{p}^{-} \geq p^{-}$is clear.

Denote by $\|\cdot\|$ the maximum norm and by $\mathbb{S}^{m}=\left\{\boldsymbol{x} \in \mathbb{R}^{m+1}:\|\boldsymbol{x}\|=1\right\}$ the unit sphere with respect to the maximum norm in $\mathbb{R}^{m+1}$. Recall that a random vector $\boldsymbol{Y} \in \mathbb{R}^{m+1}$ is multivariate regularly varying with index $-\kappa<0$ (sometimes also termed with index $\kappa>0$ ) if there exists a random vector $\boldsymbol{\Theta}$ with values in $\mathbb{S}^{m}$ such that, for every $x>0$, the measures

$$
\frac{\mathrm{P}(\|\boldsymbol{Y}\|>u x, \boldsymbol{Y} /\|\boldsymbol{Y}\| \in \cdot)}{\mathrm{P}(\|\boldsymbol{Y}\|>u)}
$$


on $\mathcal{B}\left(\mathbb{S}^{m}\right)$ converge weakly to a measure $x^{-\kappa} \mathrm{P}(\boldsymbol{\Theta} \in \cdot)$ as $u \rightarrow \infty$. The distribution of $\boldsymbol{\Theta}$ is called the spectral measure of $\boldsymbol{Y}$ (with respect to the maximum norm). Multivariate regular variation can be defined with respect to any other norm on $\mathbb{R}^{m+1}$, but since all these definitions are equivalent (only the form of the spectral measure differs), we have chosen to work with the maximum norm, which is particularly convenient for our calculations. It is further known that a random vector $\boldsymbol{Y}$ is regularly varying with index $-\kappa$ if and only if there exists a nonzero Radon measure $\sigma$ on $\overline{\mathbb{R}}^{m+1} \backslash\{\boldsymbol{0}\}$ with $\sigma\left(\overline{\mathbb{R}}^{m+1} \backslash \mathbb{R}^{m+1}\right)=0$ and a sequence $\left(a_{n}\right)_{n \in \mathbb{N}}$ of positive numbers increasing to $\infty$ such that

$$
n \mathrm{P}\left(a_{n}^{-1} \boldsymbol{Y} \in \cdot\right) \stackrel{\mathrm{v}}{\rightarrow} \sigma(\cdot) \quad \text { as } n \rightarrow \infty
$$

where ' $\stackrel{\mathrm{v}}{\rightarrow}$ ' denotes vague convergence on $\mathscr{B}\left(\overline{\mathbb{R}}^{m+1} \backslash\{\mathbf{0}\}\right)$. For further information regarding multivariate regular variation, we refer the reader to [2] or [23].

Using Lemma 3.2, we will prove that the finite-dimensional distributions

$$
\boldsymbol{X}^{(m)}=\left(X_{0}, X_{1}, \ldots, X_{m}\right)^{\top} \in \mathbb{R}^{m+1}
$$

of the stationary $\operatorname{TAR}(S, 1)$ process (3.9) are multivariate regularly varying for every $m \in \mathbb{N}_{0}$. For this, we need the definition of the following matrices: let

$$
\boldsymbol{I}=\left(\begin{array}{ll}
1 & 0 \\
0 & 1
\end{array}\right), \quad \boldsymbol{B}=\left(\begin{array}{cc}
\beta_{2}^{+} & \beta_{1}^{-} \\
\beta_{2}^{-} & \beta_{1}^{+}
\end{array}\right) \in \mathbb{R}^{2 \times 2},
$$

and, for $m \in \mathbb{N}_{0}$, define

$$
\boldsymbol{C}^{(m)}=\left(\begin{array}{ccccc}
\boldsymbol{I} & \mathbf{0} & \mathbf{0} & \cdots & \mathbf{0} \\
\boldsymbol{B} & \boldsymbol{I} & \mathbf{0} & \ddots & \vdots \\
\boldsymbol{B}^{2} & \boldsymbol{B} & \boldsymbol{I} & \ddots & \vdots \\
\vdots & \vdots & \ddots & \ddots & \mathbf{0} \\
\boldsymbol{B}^{m} & \boldsymbol{B}^{m-1} & \cdots & \boldsymbol{B} & \boldsymbol{I}
\end{array}\right), \quad \boldsymbol{S}^{(m)}=\left(\begin{array}{cccccc}
1 & -1 & 0 & 0 & \cdots & 0 \\
0 & 0 & 1 & -1 & & 0 \\
\vdots & \vdots & \ddots & \ddots & \ddots & \vdots \\
0 & 0 & \cdots & 0 & 1 & -1
\end{array}\right),
$$

where $\boldsymbol{C}^{(m)} \in \mathbb{R}^{2(m+1) \times 2(m+1)}$ and $\boldsymbol{S}^{(m)} \in \mathbb{R}^{(m+1) \times 2(m+1)}$, and $\boldsymbol{B}^{m}$ denotes the $m$ th power of $\boldsymbol{B}$ (with $\boldsymbol{B}^{0}=\boldsymbol{I}$ ). Finally, define

$$
\tilde{\boldsymbol{C}}^{(m)}:=\boldsymbol{S}^{(m)} \boldsymbol{C}^{(m)}=:\left(\boldsymbol{c}_{0}^{+}, \boldsymbol{c}_{0}^{-}, \ldots, \boldsymbol{c}_{m}^{+}, \boldsymbol{c}_{m}^{-}\right) \in \mathbb{R}^{(m+1) \times 2(m+1)} .
$$

The vectors $\boldsymbol{c}_{k}^{+}$and $\boldsymbol{c}_{k}^{-}(k=0, \ldots, m)$ will be used to describe the spectral measure of $\left(X_{0}, \ldots, X_{m}\right)^{\top}$. Insight into their structure can be obtained by writing

$$
\boldsymbol{B}^{m}=\left(\begin{array}{ll}
b_{11}^{(m)} & b_{12}^{(m)} \\
b_{21}^{(m)} & b_{22}^{(m)}
\end{array}\right) \in \mathbb{R}^{2 \times 2} \quad \text { for } m \in \mathbb{N}_{0} .
$$

Then $b_{i j}^{(m)} \geq 0$ for $m \in \mathbb{N}_{0}, i, j=1,2$, and $\boldsymbol{B}^{m}$ has at most one nonzero element in every column. Setting

$$
b_{1}^{(m)}:=b_{11}^{(m)}-b_{21}^{(m)} \quad \text { and } \quad b_{2}^{(m)}:=b_{12}^{(m)}-b_{22}^{(m)} \quad \text { for } m \in \mathbb{N}_{0},
$$


we see that the $j$ th component $\left(c_{k}^{ \pm}\right)_{j}$ of $c_{k}^{ \pm}$satisfies

$$
\begin{aligned}
& \left(c_{k}^{+}\right)_{j}=0 \quad \text { for } j \leq k, \quad\left(c_{k}^{+}\right)_{j}=b_{1}^{(j-k-1)} \text { for } k+1 \leq j \leq m+1, \\
& \left(c_{k}^{-}\right)_{j}=0 \quad \text { for } j \leq k, \quad\left(c_{k}^{-}\right)_{j}=b_{2}^{(j-k-1)} \quad \text { for } k+1 \leq j \leq m+1 \text {. }
\end{aligned}
$$

It is easy to check that $\left(\left|b_{1}^{(m)}\right|\right)_{m \in \mathbb{N}_{0}}$ and $\left(\left|b_{2}^{(m)}\right|\right)_{m \in \mathbb{N}_{0}}$ are decreasing sequences, and that $\left|b_{1}^{(m)}\right| \leq \beta^{m}$ and $\left|b_{2}^{(m)}\right| \leq \beta^{m}$. In particular, it follows that $\boldsymbol{c}_{k}^{ \pm} \in \mathbb{S}^{m}$ for $0 \leq k \leq m$. With these preparations, we can now show that the stationary version of the $\operatorname{TAR}(S, 1)$ model (3.9) is multivariate regularly varying.

Theorem 3.1. (Multivariate regular variation.) Suppose that Conditions 2.1 and 2.2 hold, and let $\left(X_{k}\right)_{k \in \mathbb{N}_{0}}$ be a stationary version of the TAR(S, 1) process as given in (3.9). Suppose further that $\left|Z_{1}\right| \in \mathcal{R}_{-\kappa}$ for some $\kappa>0$. Then $\boldsymbol{X}^{(m)}=\left(X_{0}, \ldots, X_{m}\right)^{\top}$ is multivariate regularly varying with index $-\kappa$, and its spectral measure with respect to the maximum norm is given by

$$
\mathrm{P}\left(\boldsymbol{\Theta}^{(m)} \in \cdot\right)=\frac{1}{\tilde{p}^{+}+\tilde{p}^{-}+m}\left(\tilde{p}^{+} \mathbf{1}_{\left\{c_{0}^{+} \in \cdot\right\}}+\tilde{p}^{-} \mathbf{1}_{\left\{\boldsymbol{c}_{0}^{-} \in \cdot\right\}}+\sum_{j=1}^{m}\left(p^{+} \mathbf{1}_{\left\{c_{j}^{+} \in \cdot\right\}}+p^{-} \mathbf{1}_{\left\{\boldsymbol{c}_{j}^{-} \in \cdot\right\}}\right)\right),
$$

where $\tilde{p}^{+}$and $\tilde{p}^{-}$are defined as in Lemma 3.2, and $\boldsymbol{c}_{j}^{ \pm}(j=0, \ldots, m)$ is defined as above.

Proof. We define $Z^{(m)}=\left(X_{0}^{+}, X_{0}^{-}, Z_{1}^{+}, Z_{1}^{-}, \ldots, Z_{m}^{+}, Z_{m}^{-}\right)^{\top} \in \mathbb{R}^{2(m+1)}$ for $m \in \mathbb{N}_{0}$, which, by Lemma 3.2, is multivariate regularly varying of index $-\kappa$ with spectral measure

$$
\mathrm{P}\left(\tilde{\boldsymbol{\Theta}}^{(m)} \in \cdot\right)=\frac{1}{\tilde{p}^{+}+\tilde{p}^{-}+m}\left(\tilde{p}^{+} \mathbf{1}_{\left\{\boldsymbol{e}_{1} \in \cdot\right\}}+\tilde{p}^{-} \mathbf{1}_{\left\{\boldsymbol{e}_{2} \in \cdot\right\}}+\sum_{j=1}^{m}\left(p^{+} \mathbf{1}_{\left\{\boldsymbol{e}_{2 j+1} \in \cdot\right\}}+p^{-} \mathbf{1}_{\left\{\boldsymbol{e}_{2 j+2} \in \cdot\right\}}\right)\right),
$$

where $\boldsymbol{e}_{j} \in \mathbb{R}^{2(m+1)}, j=1, \ldots, 2(m+1)$, is the unit vector with 1 in the $j$ th component and Os elsewhere.

Furthermore, we define $\left(Y_{k}\right)_{k \in \mathbb{N}_{0}}$ by $Y_{0}:=X_{0}$ and

$$
Y_{k}=\beta_{2} Y_{k-1} \mathbf{1}_{\left\{Y_{k-1}>0\right\}}+\beta_{1} Y_{k-1} \mathbf{1}_{\left\{Y_{k-1}<0\right\}}+Z_{k}=\beta_{2} Y_{k-1}^{+}-\beta_{1} Y_{k-1}^{-}+Z_{k}, \quad k \in \mathbb{N} .
$$

Let

$$
\begin{array}{ll}
\tilde{\boldsymbol{W}}:=\tilde{\boldsymbol{C}}^{(m)} \boldsymbol{Z}^{(m)}=:\left(\tilde{W}_{0}, \ldots, \tilde{W}_{m}\right)^{\top}, & \tilde{\boldsymbol{Y}}:=\left(Y_{0}, \ldots, Y_{m}\right)^{\top}, \\
\boldsymbol{W}:=\boldsymbol{C}^{(m)} \boldsymbol{Z}^{(m)}=:\left(W_{0}^{+}, W_{0}^{-}, \ldots, W_{m}^{+}, W_{m}^{-}\right)^{\top}, & \boldsymbol{Y}:=\left(Y_{0}^{+}, Y_{0}^{-}, \ldots, Y_{m}^{+}, Y_{m}^{-}\right)^{\top} .
\end{array}
$$

Since $\tilde{\boldsymbol{W}}$ is obtained from $\boldsymbol{Z}^{(m)}$ by a linear transformation, it is easy to see that $\tilde{\boldsymbol{W}}$ is again multivariate regularly varying with spectral measure $\boldsymbol{\Theta}^{(m)}$ as given in (3.23); see, e.g. [2, Proposition A.1] and [14, Lemma 2.1]. We will show that $\tilde{\boldsymbol{Y}}$ is regularly varying with the same spectral measure as $\tilde{\boldsymbol{W}}$. Furthermore, observe that, by the definition of the matrix $\boldsymbol{C}^{(m)}$, it holds that

$$
\begin{gathered}
W_{0}^{+}=X_{0}^{+}, \quad W_{0}^{-}=X_{0}^{-}, \quad W_{k+1}^{+}=\beta_{2}^{+} W_{k}^{+}+\beta_{1}^{-} W_{k}^{-}+Z_{k+1}^{+}, \\
\text {and } \quad W_{k+1}^{-}=\beta_{2}^{-} W_{k}^{+}+\beta_{1}^{+} W_{k}^{-}+Z_{k+1}^{-}, \quad \text { for } k=0, \ldots, m-1 .
\end{gathered}
$$


Let $\left(a_{n}\right)_{n \in \mathbb{N}}$ be a sequence of positive numbers increasing to $\infty$ such that $\lim _{n \rightarrow \infty} n \mathrm{P}\left(\left|Z_{1}\right|>\right.$ $\left.a_{n}\right)=1$, and let $0<\delta<1$. Then

$$
\begin{aligned}
\mathrm{P}\left(\|\tilde{\boldsymbol{W}}-\tilde{\boldsymbol{Y}}\|>2 \delta a_{n}\right) & \leq \mathrm{P}\left(\|\boldsymbol{W}-\boldsymbol{Y}\|>\delta a_{n}\right) \\
& \leq \sum_{k=1}^{m}\left[\mathrm{P}\left(\left|W_{k}^{+}-Y_{k}^{+}\right|>\delta a_{n}\right)+\mathrm{P}\left(\left|W_{k}^{-}-Y_{k}^{-}\right|>\delta a_{n}\right)\right] .
\end{aligned}
$$

Let $\Delta_{k}^{ \pm}:=W_{k}^{ \pm}-Y_{k}^{ \pm}$for $k \in \mathbb{N}_{0}$. First, we will show that

$$
\mathrm{P}\left(\left|\Delta_{1}^{+}\right|>\delta a_{n}\right)=o\left(\mathrm{P}\left(\left|Z_{1}\right|>a_{n}\right)\right) \quad \text { as } n \rightarrow \infty
$$

for any $\delta>0$, and, hence, by symmetry, the same arguments lead to

$$
\mathrm{P}\left(\left|\Delta_{1}^{-}\right|>\delta a_{n}\right)=o\left(\mathrm{P}\left(\left|Z_{1}\right|>a_{n}\right)\right) \quad \text { as } n \rightarrow \infty .
$$

Then we use induction to prove that, for any $\delta>0$,

$$
\mathrm{P}\left(\left|\Delta_{k}^{ \pm}\right|>\delta a_{n}\right)=o\left(\mathrm{P}\left(\left|Z_{1}\right|>a_{n}\right)\right) \quad \text { as } n \rightarrow \infty, k \in \mathbb{N} .
$$

Since the result is trivial if $\beta=0$, i.e. $\beta_{1}=\beta_{2}=0$, we will assume that $\beta \neq 0$ from now on. In order to study (3.25), let $0<\beta \delta_{2}<\delta_{1}<\delta$. Then

$$
\begin{aligned}
\mathrm{P}\left(\left|\Delta_{1}^{+}\right|>\delta a_{n}\right) \leq & \mathrm{P}\left(\left|\Delta_{1}^{+}\right|>\delta a_{n}, Z_{1}>\delta_{1} a_{n},\left|Y_{0}\right| \leq \delta_{2} a_{n}\right) \\
& +\mathrm{P}\left(\left|\Delta_{1}^{+}\right|>\delta a_{n}, Z_{1}<-\delta_{1} a_{n},\left|Y_{0}\right| \leq \delta_{2} a_{n}\right) \\
& +\mathrm{P}\left(\left|\Delta_{1}^{+}\right|>\delta a_{n},\left|Z_{1}\right| \leq \delta_{1} a_{n}, Y_{0}>\delta_{2} a_{n}\right) \\
& +\mathrm{P}\left(\left|\Delta_{1}^{+}\right|>\delta a_{n},\left|Z_{1}\right| \leq \delta_{1} a_{n}, Y_{0}<-\delta_{2} a_{n}\right) \\
& +\mathrm{P}\left(\left|\Delta_{1}^{+}\right|>\delta a_{n},\left|Z_{1}\right| \leq \delta_{1} a_{n},\left|Y_{0}\right| \leq \delta_{2} a_{n}\right) \\
& +\mathrm{P}\left(\left|Z_{1}\right|>\delta 1 a_{n},\left|Y_{0}\right|>\delta_{2} a_{n}\right) \\
= & I+I I+I I I+I V+V+V I, \quad \text { say. }
\end{aligned}
$$

The summand $I$ can be estimated by

$$
I \leq \mathrm{P}\left(\beta\left|Y_{0}\right|>\delta a_{n}, Z_{1}>\delta_{1} a_{n},\left|Y_{0}\right| \leq \delta_{2} a_{n}\right)=0,
$$

since $\beta \delta_{2}<\delta$. Similarly, we obtain

$$
I I \leq \mathrm{P}\left(\beta\left|Y_{0}\right|>\delta a_{n},\left|Y_{0}\right| \leq \delta_{2} a_{n}\right)=0 .
$$

That $I I I=0$ also can be seen from

$$
\begin{aligned}
I I I \leq & \mathrm{P}\left(\left|\Delta_{1}^{+}\right|>\delta a_{n},\left|Z_{1}\right| \leq \delta_{1} a_{n},\left|\beta_{2}\right| Y_{0}>\delta_{1} a_{n}\right) \\
& +\mathrm{P}\left(\left|\Delta_{1}^{+}\right|>\delta a_{n},\left|Z_{1}\right| \leq \delta_{1} a_{n}, \delta_{2}\left|\beta_{2}\right| a_{n}<\left|\beta_{2}\right| Y_{0} \leq \delta_{1} a_{n}\right) \\
= & 0+0 \\
= & 0
\end{aligned}
$$

if $\beta_{2} \neq 0$, and from $W_{1}^{+}=Y_{1}^{+}$if $\beta_{2}=0$ and $Y_{0}>0$. By symmetry, we also have

$$
I V=0 .
$$


Provided that $\delta_{1}$ and $\delta_{2}$ are small, we further have

$$
V=0
$$

and, finally, we estimate

$$
V I=\mathrm{P}\left(\left|Z_{1}\right|>\delta_{1} a_{n}\right) \mathrm{P}\left(\left|Y_{0}\right|>\delta_{2} a_{n}\right)=o\left(\mathrm{P}\left(\left|Z_{1}\right|>a_{n}\right)\right) \quad \text { as } n \rightarrow \infty .
$$

Hence, (3.27)-(3.33) give

$$
\mathrm{P}\left(\left|\Delta_{1}^{+}\right|>\delta a_{n}\right)=o\left(\mathrm{P}\left(\left|Z_{1}\right|>a_{n}\right)\right) \quad \text { as } n \rightarrow \infty .
$$

Next, we assume that (3.26) holds for some $k \in \mathbb{N}$ and every $\delta>0$. Define $\bar{W}_{k+1}^{+}:=$ $\beta_{2}^{+} Y_{k}^{+}+\beta_{1}^{-} Y_{k}^{-}+Z_{k+1}^{+}$for $k \in \mathbb{N}_{0}$, and let $\delta_{3} \in(\beta, 1)$. Then

$$
\begin{aligned}
\mathrm{P}\left(\left|\Delta_{k+1}^{+}\right|>\delta a_{n}\right) \leq & \mathrm{P}\left(\left|\Delta_{k}^{+}\right| \leq \frac{\delta a_{n}}{2},\left|\Delta_{k}^{-}\right| \leq \frac{\delta a_{n}}{2},\left|\bar{W}_{k+1}^{+}-Y_{k+1}^{+}\right|>\left(1-\delta_{3}\right) \delta a_{n}\right) \\
& +\mathrm{P}\left(\left|\Delta_{k}^{+}\right| \leq \frac{\delta a_{n}}{2},\left|\Delta_{k}^{-}\right| \leq \frac{\delta a_{n}}{2},\left|W_{k+1}^{+}-\bar{W}_{k+1}^{+}\right|>\delta 3 \delta a_{n}\right) \\
& +\mathrm{P}\left(\left|\Delta_{k}^{+}\right|>\frac{\delta a_{n}}{2}\right)+\mathrm{P}\left(\left|\Delta_{k}^{-}\right|>\frac{\delta a_{n}}{2}\right) \\
= & : V I I+V I I I+X I+X, \text { say. }
\end{aligned}
$$

With exactly the same reasoning that led to (3.34), we obtain

$$
V I I \leq \mathrm{P}\left(\left|\bar{W}_{k+1}^{+}-Y_{k+1}^{+}\right|>\left(1-\delta_{3}\right) \delta a_{n}\right)=o\left(\mathrm{P}\left(\left|Z_{1}\right|>a_{n}\right)\right) \quad \text { as } n \rightarrow \infty .
$$

On the other hand,

$$
V I I I=\mathrm{P}\left(\left|\Delta_{k}^{+}\right| \leq \frac{\delta a_{n}}{2},\left|\Delta_{k}^{-}\right| \leq \frac{\delta a_{n}}{2},\left|\beta_{2}^{+} \Delta_{k}^{+}+\beta_{1}^{-} \Delta_{k}^{-}\right|>\delta_{3} \delta a_{n}\right)=0 .
$$

Furthermore,

$$
X I=o\left(\mathrm{P}\left(\left|Z_{1}\right|>a_{n}\right)\right) \quad \text { and } \quad X=o\left(\mathrm{P}\left(\left|Z_{1}\right|>a_{n}\right)\right) \quad \text { as } n \rightarrow \infty,
$$

by the induction hypothesis. Equations (3.35)-(3.38) then give (3.26). Using (3.24) and (3.26), we hence obtain

$$
\mathrm{P}\left(\|\tilde{\boldsymbol{W}}-\tilde{\boldsymbol{Y}}\|>2 \delta a_{n}\right) \leq \mathrm{P}\left(\|\boldsymbol{W}-\boldsymbol{Y}\|>\delta a_{n}\right)=o\left(\mathrm{P}\left(\left|Z_{1}\right|>a_{n}\right)\right) \quad \text { as } n \rightarrow \infty .
$$

Since $\delta$ can be arbitrarily small, $\tilde{\boldsymbol{Y}}$ is multivariate regularly varying with the same spectral measure as $\tilde{\boldsymbol{W}}$. Finally, $\left\|\boldsymbol{X}^{(m)}-\tilde{\boldsymbol{Y}}\right\| \leq \sum_{k=1}^{m}\left((2 \beta)^{k} \max \left\{\left|r_{1}\right|,\left|r_{2}\right|\right\}+\alpha \sum_{j=0}^{k-1}(2 \beta)^{j}\right)$, which follows again by induction, shows that $\boldsymbol{X}^{(m)}$ is multivariate regularly varying with the same spectral measure as $\tilde{\boldsymbol{Y}}$ and, hence, as $\tilde{\boldsymbol{W}}$.

\subsection{Extremal behavior of the $\operatorname{TAR}(S, 1)$ model with specific partitions}

For a locally compact Hausdorff space $E$, we denote by $M_{P}(E)$ the space of all point measures on $E$. A point process is then a random element with values in $M_{P}(E)$, and a Poisson point process (which is a Poisson random measure) with mean measure $\vartheta$ will be denoted 
by $\operatorname{PRM}(\vartheta)$. In extreme value theory, the space $E$ is often $\overline{\mathbb{R}} \backslash\{0\}, \mathbb{R},[0, \infty) \times(\overline{\mathbb{R}} \backslash\{0\})$, or $[0, \infty) \times \mathbb{R}$, and the extremal behavior of a stationary sequence $\left(\xi_{k}\right)_{k \in \mathbb{N}}$ is described by the weak limit of the point process $\sum_{k=1}^{\infty} \varepsilon_{a_{n}^{-1}\left(\xi_{k}-b_{n}\right)}$ in $M_{P}(\overline{\mathbb{R}} \backslash\{0\})$ or $M_{P}(\mathbb{R})$ as $n \rightarrow \infty$ (depending on the tail behavior of $\xi_{1}$ ), or still more informatively by the weak limit of the point process $\sum_{k=1}^{\infty} \varepsilon_{\left(k / n, a_{n}^{-1}\left(\xi_{k}-b_{n}\right)\right)}$ in $M_{P}([0, \infty) \times(\overline{\mathbb{R}} \backslash\{0\}))$ or $M_{P}([0, \infty) \times \mathbb{R})$, respectively, as $n \rightarrow \infty$. Here, $a_{n}>0$ and $b_{n} \in \mathbb{R}$ are the norming constants of an associated i.i.d. sequence $\left(\tilde{\xi}_{k}\right)_{k \in \mathbb{N}}$ with distribution $\tilde{\xi}_{1} \stackrel{\text { D }}{=} \xi_{1}$ (where $\stackrel{\text { D }}{=}$, denotes equality in distribution), such that

$$
\exp \left(-\lim _{n \rightarrow \infty} n \mathrm{P}\left(\xi_{1}>a_{n} x+b_{n}\right)\right)=\lim _{n \rightarrow \infty} \mathrm{P}\left(a_{n}^{-1}\left(\bigvee_{k=1}^{n} \tilde{\xi}_{k}-b_{n}\right) \leq x\right)=G(x)
$$

for $x$ in the support of $G$, where $G$ is an extreme value distribution, i.e. either a Fréchet distribution, a Gumbel distribution, or a Weibull distribution. For further information about extreme value theory and point processes, we refer the reader to [23] and [25]. Observe in particular that (3.39) holds for $G$ being the Fréchet distribution $\Phi_{\kappa}(x)=\exp \left(-x^{-\kappa}\right) \mathbf{1}_{(0, \infty)}(x)$ with $\kappa>0$ if and only if $\xi_{1}$ is in $\mathcal{R}_{-\kappa}$, in which case the norming constants $b_{n}$ can be chosen to be 0 and $a_{n}$ can be chosen subject to $\lim _{n \rightarrow \infty} n \mathrm{P}\left(\xi_{1}>a_{n}\right)=1$.

Now we describe the extremal behavior of the stationary $\operatorname{TAR}(S, 1)$ model (3.9) via point processes. Observe that the constants $a_{n}$ defined in (3.40), below, are the norming constants of an i.i.d. sequence with the same distribution as $\left|X_{0}\right|$, rather than that of $X_{0}$.

Theorem 3.2. (Point process behavior.) Suppose that Conditions 2.1 and 2.2 hold, and let $\left(X_{k}\right)_{k \in \mathbb{N}_{0}}$ be a stationary version of the $\operatorname{TAR}(S, 1)$ process as given in (3.9). Suppose further that $\left|Z_{1}\right| \in \mathcal{R}_{-\kappa}$ for some $\kappa>0$. Let $0<a_{n} \uparrow \infty$ be a sequence of constants such that

$$
\lim _{n \rightarrow \infty} n \mathrm{P}\left(\left|X_{0}\right|>a_{n}\right)=1
$$

Then, as $n \rightarrow \infty$,

$$
\sum_{k=1}^{\infty} \varepsilon_{\left(k / n, a_{n}^{-1} X_{k}\right)} \stackrel{\mathrm{w}}{\rightarrow} \sum_{k=1}^{\infty} \sum_{j=0}^{\infty} \varepsilon_{\left(s_{k}, b_{1}^{(j)} P_{k}^{+}+b_{2}^{(j)} P_{k}^{-}\right)} \quad \text { in } M_{P}([0, \infty) \times(\overline{\mathbb{R}} \backslash\{0\})),
$$

where $b_{1}^{(j)}$ and $b_{2}^{(j)}$ are given by (3.22), $\sum_{k=1}^{\infty} \varepsilon_{\left(s_{k}, P_{k}\right)}$ is $\operatorname{PRM}(\vartheta)$ in $M_{P}([0, \infty) \times(\overline{\mathbb{R}} \backslash\{0\}))$ with

$$
\vartheta(\mathrm{d} t \times \mathrm{d} x)=\mathrm{d} t \times \kappa \theta\left(p^{+} x^{-\kappa-1} \mathbf{1}_{(0, \infty)}(x)+p^{-}(-x)^{-\kappa-1} \mathbf{1}_{(-\infty, 0)}(x)\right) \mathrm{d} x
$$

and

$$
\theta=\left(p^{+} \sum_{j=0}^{\infty}\left|b_{1}^{(j)}\right|^{\kappa}+p^{-} \sum_{j=0}^{\infty}\left|b_{2}^{(j)}\right|^{\kappa}\right)^{-1}=\left(\tilde{p}^{+}+\tilde{p}^{-}\right)^{-1} \in(0,1] .
$$

Here, $\tilde{p}^{+}$and $\tilde{p}^{-}$are given by (3.10) and (3.11), respectively.

Proof. We apply the results of [7, Theorem 2.7]. By Lemma 2.1, $\left(X_{k}\right)_{k \in \mathbb{N}_{0}}$ is geometrically strongly mixing. Hence, the mixing condition $\mathcal{A}\left(a_{n}\right)$ of $[7$, p. 882] is satisfied, meaning that there exists a sequence of positive integers $\left(v_{n}\right)_{n \in \mathbb{N}}$ such that $\lim _{n \rightarrow \infty} v_{n}=\infty, \lim _{n \rightarrow \infty} v_{n} / n=$ 0 , and

$$
\lim _{n \rightarrow \infty} \operatorname{E} \exp \left(-\sum_{j=1}^{n} f\left(\frac{X_{j}}{a_{n}}\right)\right)-\left(\operatorname{Eexp}\left(-\sum_{j=1}^{v_{n}} f\left(\frac{X_{j}}{a_{n}}\right)\right)\right)^{\left\lfloor n / v_{n}\right\rfloor}=0
$$


holds for all step functions $f$ on $\overline{\mathbb{R}} \backslash\{0\}$ with bounded support which is bounded away from 0 . Furthermore, for $x>0$,

$$
\begin{aligned}
& \mathrm{P}\left(\bigvee_{m \leq k \leq v_{n}}\left|X_{k}\right|>x a_{n},\left|X_{0}\right|>x a_{n}\right) \\
& \quad \leq \sum_{m \leq k \leq v_{n}} \mathrm{P}\left(\left|X_{k}\right|>x a_{n},\left|X_{0}\right|>x a_{n}\right) \\
& \quad \leq \sum_{m \leq k \leq v_{n}} \mathrm{P}\left(\beta^{k}\left|X_{0}\right|+\alpha \sum_{j=0}^{k-1} \beta^{j}+\sum_{j=1}^{k} \beta^{k-j}\left|Z_{j}\right|>x a_{n},\left|X_{0}\right|>x a_{n}\right) \\
& \quad \leq \sum_{m \leq k \leq v_{n}} \mathrm{P}\left(\beta^{k}\left|X_{0}\right|+\frac{\alpha}{1-\beta}+\sum_{j=0}^{\infty} \beta^{j}\left|Z_{j}\right|>x a_{n},\left|X_{0}\right|>x a_{n}\right) .
\end{aligned}
$$

Let $\delta \in(0, x)$. Then (3.41) and the independence of $X_{0}$ and $\left(Z_{k}\right)_{k \in \mathbb{N}_{0}}$ result in

$$
\begin{aligned}
& \mathrm{P}\left(\bigvee_{m \leq k \leq v_{n}}\left|X_{k}\right|>x a_{n},\left|X_{0}\right|>x a_{n}\right) \\
& \leq \sum_{m \leq k \leq v_{n}} \mathrm{P}\left(\beta^{k}\left|X_{0}\right|+\frac{\alpha}{1-\beta}>\delta a_{n}\right) \\
& \quad+\sum_{m \leq k \leq v_{n}} \mathrm{P}\left(\sum_{j=0}^{\infty} \beta^{j}\left|Z_{j}\right|>(x-\delta) a_{n}\right) \mathrm{P}\left(\left|X_{0}\right|>x a_{n}\right) \\
& =: J_{1}(n)+J_{2}(n), \quad \text { say. }
\end{aligned}
$$

Since $\left|X_{0}\right| \in \mathcal{R}_{-\kappa}$ by Lemma 3.2, and since the limit in (3.1) is uniform for $u \in\left[u_{0}, \infty\right)$ for every $u_{0}>0$ (see, e.g. [23, Proposition 0.5$]$ ), it follows using dominated convergence that

$$
\limsup _{m \rightarrow \infty} \lim _{n \rightarrow \infty} \frac{J_{1}(n)}{\mathrm{P}\left(\left|X_{0}\right|>x a_{n}\right)} \leq \limsup _{m \rightarrow \infty} \sum_{k \geq m}\left(\frac{\beta^{-k} \delta}{x}\right)^{-\kappa} \leq \limsup _{m \rightarrow \infty}\left(\frac{\delta}{x}\right)^{-\kappa} \sum_{k \geq m}\left(\beta^{\kappa}\right)^{k}=0 .
$$

We can estimate $J_{2}(n)$ by

$$
\limsup _{m \rightarrow \infty} \lim _{n \rightarrow \infty} \frac{J_{2}(n)}{\mathrm{P}\left(\left|X_{0}\right|>x a_{n}\right)} \leq \limsup _{m \rightarrow \infty} \lim _{n \rightarrow \infty} v_{n} \mathrm{P}\left(\sum_{j=0}^{\infty} \beta^{j}\left|Z_{j}\right|>(x-\delta) a_{n}\right)=0,
$$

since $\lim _{n \rightarrow \infty} v_{n} / n=0$ and since

$$
\begin{aligned}
\mathrm{P}\left(\left|X_{0}\right|>x\right) & \sim\left(\tilde{p}^{+}+\tilde{p}^{-}\right) \mathrm{P}\left(\left|Z_{1}\right|>x\right) \\
& \sim\left(\tilde{p}^{+}+\tilde{p}^{-}\right)\left(1-\beta^{\kappa}\right) \mathrm{P}\left(\sum_{j=0}^{\infty} \beta^{j}\left|Z_{j}\right|>x\right) \text { as } x \rightarrow \infty .
\end{aligned}
$$

Hence, we conclude that

$$
\lim _{m \rightarrow \infty} \lim _{n \rightarrow \infty} \mathrm{P}\left(\bigvee_{m \leq k \leq v_{n}}\left|X_{k}\right|>x a_{n}|| X_{0} \mid>x a_{n}\right)=0, \quad x>0
$$


which is Equation (2.8) of [7]. Since $\boldsymbol{X}^{(2 m+1)}$ is multivariate regularly varying with index $-\kappa$ and spectral measure described by the vector $\Theta^{(2 m)}=\left(\Theta_{j}^{(2 m)}\right)_{j=0, \ldots, 2 m}$ as given in Theorem 3.1, we obtain

$\mathrm{E}\left|\Theta_{m}^{(2 m)}\right|^{\kappa}=\frac{1}{\tilde{p}^{+}+\tilde{p}^{-}+2 m}\left(\tilde{p}^{+}\left|b_{1}^{(m)}\right|^{\kappa}+\tilde{p}^{-}\left|b_{2}^{(m)}\right|^{\kappa}+p^{+} \sum_{j=0}^{m-1}\left|b_{1}^{(j)}\right|^{\kappa}+p^{-} \sum_{j=0}^{m-1}\left|b_{2}^{(j)}\right|^{\kappa}\right)$.

Using further that $\left(\left|b_{1}^{(m)}\right|\right)_{m \geq 0}$ and $\left(\left|b_{2}^{(m)}\right|\right)_{m \geq 0}$ are nonincreasing sequences, we obtain

$$
\begin{aligned}
& \mathrm{E}\left(\bigvee_{k=m}^{2 m}\left|\Theta_{k}^{(2 m)}\right|^{\kappa}-\bigvee_{k=m+1}^{2 m}\left|\Theta_{k}^{(2 m)}\right|^{\kappa}\right) \\
& =\frac{1}{\tilde{p}^{+}+\tilde{p}^{-}+2 m}\left[\tilde{p}^{+}\left(\left|b_{1}^{(m)}\right|^{\kappa}-\left|b_{1}^{(m+1)}\right|^{\kappa}\right)+\tilde{p}^{-}\left(\left|b_{2}^{(m)}\right|^{\kappa}-\left|b_{2}^{(m+1)}\right|^{\kappa}\right)\right. \\
& \left.\quad+p^{+} \sum_{j=0}^{m-1}\left(\left|b_{1}^{(j)}\right|^{\kappa}-\left|b_{1}^{(j+1)}\right|^{\kappa}\right)+p^{-} \sum_{j=0}^{m-1}\left(\left|b_{2}^{(j)}\right|^{\kappa}-\left|b_{2}^{(j+1)}\right|^{\kappa}\right)\right] \\
& =\frac{1-p^{+}\left|b_{1}^{(m)}\right|^{\kappa}-p^{-}\left|b_{2}^{(m)}\right|^{\kappa}+\tilde{p}^{+}\left(\left|b_{1}^{(m)}\right|^{\kappa}-\left|b_{1}^{(m+1)}\right|^{\kappa}\right)+\tilde{p}^{-}\left(\left|b_{2}^{(m)}\right|^{\kappa}-\left|b_{2}^{(m+1)}\right|^{\kappa}\right)}{\tilde{p}^{+}+\tilde{p}^{-}+2 m} .
\end{aligned}
$$

Then

$$
\lim _{m \rightarrow \infty} \frac{\mathrm{E}\left(\bigvee_{k=m}^{2 m}\left|\Theta_{k}^{(2 m)}\right|^{\kappa}-\bigvee_{k=m+1}^{2 m}\left|\Theta_{k}^{(2 m)}\right|^{\kappa}\right)}{\mathrm{E}\left|\Theta_{m}^{2 m}\right|^{\kappa}}=\frac{1}{p^{+} \sum_{j=0}^{\infty}\left|b_{1}^{(j)}\right|^{\kappa}+p^{-} \sum_{j=0}^{\infty}\left|b_{2}^{(j)}\right|^{\kappa}}=\theta,
$$

where $\theta \in\left(1-\beta^{\kappa}, 1\right]$, since $\left|b_{1}^{(j)}\right|,\left|b_{2}^{(j)}\right| \leq \beta^{j}$, and $\left|b_{1}^{(0)}\right|=\left|b_{2}^{(0)}\right|=1$.

Similarly, defining the probability measures $R_{m}$ on $M_{P}(\overline{\mathbb{R}} \backslash\{0\})$ by

$$
R_{m}(\cdot)=\frac{\mathrm{E}\left(\left[\bigvee_{k=m}^{2 m}\left|\Theta_{k}^{(2 m)}\right|^{\kappa}-\bigvee_{k=m+1}^{2 m}\left|\Theta_{k}^{(2 m)}\right|^{\kappa}\right] \mathbf{1}_{\left\{\sum_{j=0}^{2 m} \varepsilon_{\Theta_{j}^{(2 m)}}^{(2 m} \in \cdot\right\}}\right)}{\mathrm{E}\left(\bigvee_{k=m}^{2 m}\left|\Theta_{k}^{(2 m)}\right|^{\kappa}-\bigvee_{k=m+1}^{2 m}\left|\Theta_{k}^{(2 m)}\right|^{\kappa}\right)},
$$

it follows that $R_{m}$ converges weakly as $m \rightarrow \infty$ to the distribution of the point process $\sum_{j=0}^{\infty} \varepsilon_{Q_{j}} \in M_{P}(\overline{\mathbb{R}} \backslash\{0\})$, where

$$
\sum_{j=0}^{\infty} \varepsilon_{Q_{j}}=\chi_{1} \sum_{j=0}^{\infty} \varepsilon_{b_{1}^{(j)}}+\left(1-\chi_{1}\right) \sum_{j=0}^{\infty} \varepsilon_{b_{2}^{(j)}}
$$

with $\mathrm{P}\left(\chi_{1}=1\right)=p^{+}$and $\mathrm{P}\left(\chi_{1}=0\right)=p^{-}$. Hence, the assumptions of [7, Theorem 2.7] are all satisfied. Let $\sum_{k=1}^{\infty} \varepsilon_{\left(\tilde{s}_{k}, \tilde{P}_{k}\right)}$ be $\operatorname{PRM}(\tilde{\vartheta})$ on $[0, \infty) \times(\overline{\mathbb{R}} \backslash\{0\})$ with

$$
\tilde{\vartheta}(\mathrm{d} t \times \mathrm{d} x)=\mathrm{d} t \times \kappa \theta x^{-\kappa-1} \mathbf{1}_{(0, \infty)}(x) \mathrm{d} x,
$$

and let $\left(\sum_{j=0}^{\infty} \varepsilon_{Q_{k j}}\right)_{k \in \mathbb{N}}$ be an i.i.d. sequence with $\sum_{j=0}^{\infty} \varepsilon_{Q_{k j}} \stackrel{\mathrm{D}}{=} \sum_{j=0}^{\infty} \varepsilon_{Q_{j}}$, independent of $\sum_{k=1}^{\infty} \varepsilon_{\left(\tilde{s}_{k}, \tilde{P}_{k}\right)}$. Then Theorem 2.7, Corollary 2.4, and Remark 2.3 of [7] imply that

$$
\sum_{k=1}^{\infty} \varepsilon_{\left(k / n, a_{n}^{-1} X_{k}\right)} \stackrel{\mathrm{w}}{\rightarrow} \sum_{k=1}^{\infty} \sum_{j=0}^{\infty} \varepsilon_{\left(\tilde{s}_{k}, Q_{k j} \tilde{P}_{k}\right)} \quad \text { in } M_{P}([0, \infty) \times(\overline{\mathbb{R}} \backslash\{0\})) \quad \text { as } n \rightarrow \infty
$$


(see also Lemma 4.1.2 of [18] for the connection between the convergence of the process $\sum_{k=1}^{\infty} \varepsilon_{\left(k / n, a_{n}^{-1} X_{k}\right)}$ and that of $\sum_{k=1}^{\infty} \varepsilon_{a_{n}^{-1} X_{k}}$ as $\left.n \rightarrow \infty\right)$. Since

$$
\sum_{k=1}^{\infty} \sum_{j=0}^{\infty} \varepsilon_{\left(\tilde{s}_{k}, Q_{k j} \tilde{P}_{k}\right)} \stackrel{\mathrm{D}}{=} \sum_{k=1}^{\infty} \sum_{j=0}^{\infty} \varepsilon_{\left(s_{k}, b_{1}^{(j)} P_{k}^{+}+b_{2}^{(j)} P_{k}^{-}\right)}
$$

the result follows, apart from the representation $\theta=\left(\tilde{p}^{+}+\tilde{p}^{-}\right)^{-1}$. To see the latter, suppose, for example, that $\beta_{1} \leq 0$ and $\beta_{2}>0$. Then

$$
\tilde{p}^{+}+\tilde{p}^{-}=\frac{p^{+}+p^{-}\left(-\beta_{1}\right)^{\kappa}}{1-\beta_{2}^{\kappa}}+p^{-}=p^{+} \frac{1}{1-\beta_{2}^{\kappa}}+p^{-} \frac{1-\beta_{2}^{\kappa}+\left(-\beta_{1}\right)^{\kappa}}{1-\beta_{2}^{\kappa}} .
$$

On the other hand,

$$
b_{1}^{(j)}=\beta_{2}^{j}, \quad j \in \mathbb{N}_{0}, \quad b_{2}^{(0)}=-1, \quad b_{2}^{(j)}=\left(-\beta_{1}\right) \beta_{2}^{j-1}, \quad j \in \mathbb{N},
$$

so that

$$
p^{+} \sum_{j=0}^{\infty}\left|b_{1}^{(j)}\right|^{\kappa}+p^{-} \sum_{j=0}^{\infty}\left|b_{2}^{(j)}\right|^{\kappa}=p^{+} \frac{1}{1-\beta_{2}^{\kappa}}+p^{-} \frac{1-\beta_{2}^{\kappa}+\left(-\beta_{1}\right)^{\kappa}}{1-\beta_{2}^{\kappa}}=\tilde{p}^{+}+\tilde{p}^{-} .
$$

The other cases follow similarly.

Having the point process convergence in Theorem 3.2, it is standard to derive many results about the asymptotic behavior of the stationary sequence $\left(X_{k}\right)_{k \in \mathbb{N}_{0}}$, such as convergence of the maxima to extremal processes, the asymptotic distribution of the order statistics or of exceedances over high thresholds, or the determination of the extremal index. We will concentrate here on the latter. Recall that a stationary sequence $\left(\xi_{k}\right)_{k \in \mathbb{N}}$ has extremal index $\rho \in(0,1]$ if there exist norming constants $a_{n}>0$ and $b_{n} \in \mathbb{R}$, and a nondegenerate distribution function $G$ such that (3.39) holds for an associated i.i.d. sequence $\left(\tilde{\xi}_{k}\right)_{k \in \mathbb{N}}$ with $\tilde{\xi}_{1} \stackrel{\text { D }}{=} \xi_{1}$, and

$$
\lim _{n \rightarrow \infty} \mathrm{P}\left(a_{n}^{-1}\left(\bigvee_{k=1}^{n} \xi_{k}-b_{n}\right) \leq x\right)=G(x)^{\rho} \quad \text { for all } x \in \mathbb{R} .
$$

Under weak mixing conditions (which are satisfied in our case), it is known that the reciprocal $\rho^{-1}$ of the extremal index can be interpreted as the mean cluster size of exceedances over high thresholds. In particular, an extremal index of size 1 says that high exceedances of a stationary sequence behave asymptotically like that of an i.i.d. sequence with the same marginals, while an extremal index which is less than 1 shows that clusters occur. We refer the reader to [12] and [20] for further information regarding the extremal index.

The following result gives the asymptotic behavior of the maxima of the stationary $\operatorname{TAR}(S, 1)$ model and its extremal index.

Corollary 3.1. Let the assumptions of Theorem 3.2 hold with $\left(a_{n}\right)_{n \in \mathbb{N}}$ as defined in (3.40), and define $M_{n}=\max _{k=1, \ldots, n} X_{k}$ for $n \in \mathbb{N}$. Then, for $x>0$,

$$
\lim _{n \rightarrow \infty} \mathrm{P}\left(a_{n}^{-1} M_{n} \leq x\right)=\exp \left(-\theta\left(p^{+}+p^{-}\left(\beta_{1}^{-}\right)^{\kappa}\right) x^{-\kappa}\right)=\exp \left(-\frac{p^{+}+p^{-}\left(\beta_{1}^{-}\right)^{\kappa}}{\tilde{p}^{+}+\tilde{p}^{-}} x^{-\kappa}\right),
$$

with $\tilde{p}^{+}$and $\tilde{p}^{-}$as defined in (3.10) and (3.11). In particular, if $\tilde{p}^{+}>0$ then $\left(X_{k}\right)_{k \in \mathbb{N}_{0}}$ has extremal index $\rho=1-\left(\beta_{2}^{+}\right)^{\kappa}-\left(\beta_{1}^{-}\right)^{\kappa}\left(\beta_{2}^{-}\right)^{\kappa}$. 
Proof. Applying the continuous mapping theorem for the functional

$$
T_{1}: M_{P}([0, \infty) \times(\overline{\mathbb{R}} \backslash\{0\})) \rightarrow \overline{\mathbb{R}}, \quad \sum_{k=1}^{\infty} \varepsilon_{\left(t_{k}, j_{k}\right)} \mapsto \sup \left\{j_{k}: t_{k} \leq 1\right\},
$$

it follows from Theorem 3.2 that

$$
\begin{aligned}
a_{n}^{-1} M_{n} & \stackrel{\mathrm{w}}{\rightarrow} Y \\
& :=\sup \left\{b_{1}^{(j)} P_{k}^{+}+b_{2}^{(j)} P_{k}^{-}: s_{k} \leq 1, j \in \mathbb{N}_{0}\right\} \quad(n \rightarrow \infty) \\
& =\sup \left\{P_{k}^{+}, \beta_{1}^{-} P_{k}^{-}: s_{k} \leq 1\right\} \\
& =T_{1}(\bar{N}),
\end{aligned}
$$

where $\bar{N}:=\sum_{k=1}^{\infty} \varepsilon_{\left(s_{k}, P_{k}^{+}\right)}+\varepsilon_{\left(s_{k}, \beta_{1}^{-} P_{k}^{-}\right)}$. Here, we used the facts that $\sup \left\{0, b_{1}^{(j)}: j \in \mathbb{N}_{0}\right\}=1$ and $\sup \left\{0, b_{2}^{(j)}: j \in \mathbb{N}_{0}\right\}=\beta_{1}^{-}$. Since $\bar{N}$ is $\operatorname{PRM}(\bar{\vartheta})$ with mean measure

$$
\bar{\vartheta}(\mathrm{d} t \times \mathrm{d} x)=\mathrm{d} t \times \kappa \theta\left(p^{+}+p^{-}\left(\beta_{1}^{-}\right)^{\kappa}\right) x^{-\kappa-1} \mathbf{1}_{(0, \infty)}(x) \mathrm{d} x,
$$

we conclude that, for fixed $x>0$,

$$
\begin{aligned}
\lim _{n \rightarrow \infty} \mathrm{P}\left(a_{n}^{-1} M_{n} \leq x\right) & =\mathrm{P}(Y \leq x) \\
& =\mathrm{P}(\bar{N}((0,1] \times(x, \infty))=0) \\
& =\exp \left(-\theta\left(p^{+}+p^{-}\left(\beta_{1}^{-}\right)^{\kappa}\right) x^{-\kappa}\right) .
\end{aligned}
$$

The extremal index $\rho$ of $\left(X_{k}\right)_{k \in \mathbb{N}_{0}}$ is then given by

$$
\rho=\frac{p^{+}+p^{-}\left(\beta_{1}^{-}\right)^{\kappa}}{\tilde{p}^{+}+\tilde{p}^{-}} / \frac{\tilde{p}^{+}}{\tilde{p}^{+}+\tilde{p}^{-}}=1-\left(\beta_{2}^{+}\right)^{\kappa}-\left(\beta_{1}^{-}\right)^{\kappa}\left(\beta_{2}^{-}\right)^{\kappa},
$$

which is the claim.

We can also compute the asymptotic cluster probabilities $\mathrm{P}\left(\zeta_{1}=j\right)$ of exceedances $X_{k}>$ $a_{n} x$ of length $j \in \mathbb{N}$ for fixed $x>0$ explicitly, considering the limit behavior of the rescaled times $k / n$ for which $X_{k}>a_{n} x$. This is done using the same arguments as in [8, Section 3.D]. We omit the proof.

Corollary 3.2. Let the assumptions of Theorem 3.2 hold with $\left(a_{n}\right)_{n \in \mathbb{N}}$ as defined in (3.40), and suppose that $\tilde{p}^{+}>0$, i.e. $p^{+}+p^{-}\left(\beta_{1}^{-}\right)^{\kappa}>0$. Let $\left(\tilde{s}_{k}\right)_{k \in \mathbb{N}}$ be the jump times of a Poisson process with intensity $\theta\left(p^{+}+p^{-}\left(\beta_{1}^{-}\right)^{\kappa}\right) x^{-\kappa}, x>0$, fixed. Let $\left(\zeta_{k}\right)_{k \in \mathbb{N}}$ be an $\mathbb{N}$-valued i.i.d. sequence, independent of $\left(\tilde{s}_{k}\right)_{k \in \mathbb{N}}$, with distribution

$$
\mathrm{P}\left(\zeta_{1}=j\right)=\frac{p^{+}\left(\left(\tilde{b}_{1}^{(j-1)}\right)^{\kappa}-\left(\tilde{b}_{1}^{(j)}\right)^{\kappa}\right)+p^{-}\left(\left(\tilde{b}_{2}^{(j-1)}\right)^{\kappa}-\left(\tilde{b}_{2}^{(j)}\right)^{\kappa}\right)}{p^{+}+p^{-}\left(\beta_{1}^{-}\right)^{\kappa}} \quad \text { for } j \in \mathbb{N},
$$

where $1=\tilde{b}_{1}^{(0)} \geq \tilde{b}_{1}^{(1)} \geq \cdots$ is the order statistic of the sequence $\left(\max \left\{0, b_{1}^{(j)}\right\}\right)_{j \in \mathbb{N}_{0}}$ and $\beta_{1}^{-}=\tilde{b}_{2}^{(0)} \geq \tilde{b}_{2}^{(1)} \geq \cdots$ is the order statistic of the sequence $\left(\max \left\{0, b_{2}^{(j)}\right\}\right)_{j \in \mathbb{N}_{0}}$. Then

$$
\sum_{k=1}^{\infty} \varepsilon_{\left(k / n, a_{n}^{-1} X_{k}\right)}(\cdot \times(x, \infty)) \stackrel{\mathrm{w}}{\rightarrow} \sum_{k=1}^{\infty} \zeta_{k} \varepsilon_{\tilde{s}_{k}} \quad \text { in } M_{P}([0, \infty) \text { as } n \rightarrow \infty
$$


Remarks 3.1. (i) If $\beta_{1}=\beta_{2}=\cdots=\beta_{S}=\beta \in(-1,1)$ and $\alpha_{1}=\cdots=\alpha_{S}=0$, then process (3.9) is a causal $\mathrm{AR}(1)$ process $X_{k}=\beta X_{k-1}+Z_{k}$, which can be written as an infinite moving average process $X_{k}=\sum_{j=0}^{\infty} \beta^{j} Z_{k-j}$. It is then easy to see that the results obtained in this paper are in line with those of [8] for infinite moving average processes with regularly varying noise.

(ii) Provided that $\tilde{p}^{+}>0$, the extremal index $\rho$ of Corollary 3.1 is strictly less than 1 if and only if $\beta_{2}>0$ or $\beta_{1} \beta_{2}>0$. In these cases, the $\operatorname{TAR}(S, 1)$ model can model clusters.

(iii) The value $\theta$ is the extremal index of $\left(\left|X_{k}\right|\right)_{k \in \mathbb{N}_{0}}$.

\section{Noise with at most an exponentially decreasing tail}

In this section we are interested in noise sequences which are lighter tailed than regularly varying functions, but whose tail is still not too light. Since distributions which are not regularly varying and do not have a finite right endpoint can only be in the maximum domain of attraction of the Gumbel distribution if they are in the maximum domain of attraction of some extreme value distribution at all, we will concentrate on specific i.i.d. noise sequences $\left(Z_{k}\right)_{k \in \mathbb{N}_{0}}$ for which (3.39) holds with $G(x)=\Lambda(x)=\exp \left(-\mathrm{e}^{-x}\right)$ and $\xi_{k}=Z_{k}$, which we denote by $Z_{1} \in \operatorname{MDA}(\Lambda)$. Besides the TB condition on $Z_{1}$, we will look at distributions which are either subexponential or have a tail which is close to that of an exponential distribution. More precisely, we will look at distribution functions $F$ in the class $\mathcal{L}(\gamma)$ with $\gamma \in[0, \infty)$, i.e. distribution functions which satisfy $F(x)<1$ for all $x \in \mathbb{R}$ and for which

$$
\lim _{x \rightarrow \infty} \frac{\bar{F}(x+y)}{\bar{F}(x)}=\mathrm{e}^{-\gamma y}
$$

holds locally uniformly in $y \in \mathbb{R}$. For $\gamma>0$, this means that $F$ has a tail which is close to that of an exponential distribution. For $\gamma=0$, we need a further assumption: a distribution function $F$ (defined on $\mathbb{R}$ ) is called subexponential if $F \in \mathcal{L}(0)$ and $\lim _{x \rightarrow \infty} \overline{F * F}(x) / \bar{F}(x)=2$. The class of subexponential distributions will be denoted by $\&$. For a random variable $Z$ with distribution function $F$, we simply write $X \in \delta$ or $Z \in \mathcal{L}(\gamma)$ if $F$ has the corresponding property. Subexponential distributions and those which are in $\mathcal{L}(\gamma)$ for $\gamma>0$ are handy classes of (semi-)heavy-tailed distributions, and the extremal and the tail behaviors of infinite moving average processes associated with such noise sequences have been studied in [9] and [26], respectively. Examples of distributions in $\operatorname{MDA}(\Lambda) \cap \&$ include tails of the form $\bar{F}(x) \sim$ $\exp \left(-x /(\log x)^{a}\right), a>0$, or $\bar{F}(x) \sim K x^{b} \exp \left(-x^{p}\right)$, where $p \in(0,1), K>0$, and $b \in \mathbb{R}$ $(x \rightarrow \infty)$, or the lognormal distribution. The class of subexponential distributions also includes those which have regularly varying tails, but these are not in $\operatorname{MDA}(\Lambda)$. Examples in $\mathcal{L}(\gamma)$ with $\gamma>0$ include distribution functions with tails of the form $\bar{F}(x) \sim K x^{b} \mathrm{e}^{-\gamma x}(x \rightarrow \infty)$ with $K>0$ and $b \in \mathbb{R}$, or certain generalized inverse Gaussian distributions. Observe that $\mathcal{L}(\gamma) \subset \operatorname{MDA}(\Lambda)$ for $\gamma>0$ since, by (4.1), (3.39) holds with $a_{n}=\gamma^{-1}$ and $b_{n}=\bar{F}^{\leftarrow}(1 / n)$. Also, observe that whether a distribution function is in $\delta \cap \operatorname{MDA}(\Lambda)$ or in $\mathcal{L}(\gamma)$ with $\gamma>0$, respectively, is completely determined by its tail behavior. More precisely, if $\bar{G}(x) \sim c \bar{F}(x)$ $(x \rightarrow \infty)$ for some $c \in(0, \infty)$ then $F \in \delta \cap \operatorname{MDA}(\Lambda)$ or $F \in \mathcal{L}(\gamma)$ implies the same for $G$; see [22, Lemma 2.4] for the subexponential case (the case $\mathcal{L}(\gamma)$ follows from the definition of $\mathcal{L}(\gamma))$.

As in Section 3, we will first present the tail behavior of the TAR model if $Z$ has the described noise. However, unlike there we do not have to restrict to specific $\operatorname{TAR}(S, 1)$ models to remain in the same noise class, we can handle the general $\operatorname{TAR}(S, q)$ model. 
Proposition 4.1. (Tail behavior.) Suppose that Conditions 2.1 and 2.2 hold with $p^{+}>0$, and let $\left(X_{k}\right)_{k \in \mathbb{N}_{0}}$ be a stationary version of the $\operatorname{TAR}(S, q)$ process as given in (1.1). Suppose further that $Z_{1} \in \mathcal{L}(\gamma)$ with $\gamma>0$, or that $Z_{1} \in \delta \cap \operatorname{MDA}(\Lambda)$, in which case we set $\gamma:=0$. Then $\operatorname{E} \exp \left(\gamma\left(X_{1}-Z_{1}\right)\right)$ is finite and

$$
\mathrm{P}\left(X_{1}>x\right) \sim \mathrm{E} \exp \left(\gamma\left(X_{1}-Z_{1}\right)\right) \mathrm{P}\left(Z_{1}>x\right) \text { as } x \rightarrow \infty .
$$

In particular, if $Z_{1} \in \mathcal{L}(\gamma)$ or $Z_{1} \in \delta \cap \operatorname{MDA}(\Lambda)$, respectively, then so is $X_{1}$.

Proof. Similarly to and with the same notation as in Lemma 2.2 we obtain

$$
\mathrm{P}\left(\left|X_{k}-Z_{k}\right|>x\right) \leq \mathrm{P}\left(\left|\tilde{X}_{k}+\alpha-\tilde{Z}_{k}\right|>x\right)=\mathrm{P}\left(\sum_{j=1}^{\infty} \psi_{j} \tilde{Z}_{j}+\alpha>x\right),
$$

so that

$$
\limsup _{x \rightarrow \infty} \frac{\mathrm{P}\left(\left|X_{k}-Z_{k}\right|>x\right)}{\mathrm{P}\left(Z_{1}>x\right)} \leq \frac{1}{p^{+}} \limsup _{x \rightarrow \infty} \frac{\mathrm{P}\left(\sum_{j=1}^{\infty} \psi_{j} \tilde{Z}_{j}+\alpha>x\right)}{\mathrm{P}\left(\tilde{Z}_{1}>x\right)}
$$

by Condition 2.1. Since $0 \leq \psi_{j}<1$ for $j \in \mathbb{N}$ and since the $\left(\psi_{j}\right)$ are exponentially decreasing, it follows from Proposition 1.3 of [9] that the right-hand side of (4.4) is 0 if $Z_{1} \in \& \cap \operatorname{MDA}(\Lambda)$ and Condition 2.1 is valid, and, hence, that (4.2) holds in the case $Z_{1} \in \delta \cap \operatorname{MDA}(\Lambda)(\gamma=0)$ by [11, Proposition 1] (for subexponentials on $\mathbb{R}$, see also [22, Lemma 5.1]).

Now, suppose that $\gamma>0$ and that $Z_{1} \in \mathcal{L}(\gamma)$. Together with Condition 2.1, this implies that $\psi \tilde{Z}_{1} \in \mathcal{L}(\gamma / \psi)$ for $0<\psi<1$, so that $\operatorname{Eexp}\left(\delta \tilde{Z}_{1}\right)<\infty$ for $0 \leq \delta<\gamma$. Observe that $0 \leq \psi_{j}<1$ for $j \in \mathbb{N}$ and that $\sum_{j=1}^{\infty} \psi_{j}<\infty$. Choosing $0<\varepsilon<\gamma$ such that $(\gamma+\varepsilon)^{-1}(\gamma-\varepsilon)>\max _{j \in \mathbb{N}}\left\{\psi_{j}\right\}$, it follows, from (4.3) and Jensen's inequality, that

$$
\begin{aligned}
\operatorname{Eexp}\left((\gamma+\varepsilon)\left|X_{1}-Z_{1}\right|\right) & \leq \mathrm{e}^{(\gamma+\varepsilon) \alpha} \prod_{j=1}^{\infty} \operatorname{Eexp}\left((\gamma+\varepsilon) \psi_{j} \tilde{Z}_{j}\right) \\
& \leq \mathrm{e}^{(\gamma+\varepsilon) \alpha} \prod_{j=1}^{\infty}\left(\operatorname{Eexp}\left((\gamma-\varepsilon) \tilde{Z}_{j}\right)\right)^{(\gamma+\varepsilon) \psi_{j} /(\gamma-\varepsilon)} \\
& <\infty
\end{aligned}
$$

But, since $Z_{1} \in \mathcal{L}(\gamma)$ if and only if $\exp \left(Z_{1}\right) \in \mathcal{R}_{-\gamma}$, and since $\mathrm{E} \exp \left(\left|X_{1}-Z_{1}\right|(\gamma+\varepsilon)\right)<\infty$, it follows from Breiman's [4] result on the products of regularly varying distributions that

$$
\begin{aligned}
\mathrm{P}\left(\exp \left(X_{1}\right)>x\right) & =\mathrm{P}\left(\exp \left(Z_{1}\right) \exp \left(X_{1}-Z_{1}\right)>x\right) \\
& \sim \mathrm{E} \exp \left(\gamma\left(X_{1}-Z_{1}\right)\right) \mathrm{P}\left(\exp \left(Z_{1}\right)>x\right) \quad \text { as } x \rightarrow \infty,
\end{aligned}
$$

which is (4.2) (the latter equation can also be derived from [9, Proposition 1.1] and [22, Lemma 2.1]).

Similarly to the regularly varying TAR $(S, 1)$ model, the tail of the TAR process is equivalent to the tail of the noise. Next, analogously to the regularly varying case, we show the convergence of a sequence of point processes. In contrast to Theorem 3.2 we obtain the convergence to a Poisson random measure. Thus, this model cannot exhibit extremal clusters. 
Theorem 4.1. (Point process behavior.) Suppose that Conditions 2.1 and 2.2 hold with $p^{+}>0$, and let $\left(X_{k}\right)_{k \in \mathbb{N}_{0}}$ be a stationary version of the $\operatorname{TAR}(S, q)$ process as given in (1.1). Suppose further that $Z_{1} \in \mathcal{L}(\gamma)$ with $\gamma>0$, or that $Z_{1} \in \delta \cap \operatorname{MDA}(\Lambda)$. Let $a_{n}>0$ and $b_{n} \in \mathbb{R}$ be sequences of constants such that

$$
\lim _{n \rightarrow \infty} n \mathrm{P}\left(X_{0}>a_{n} x+b_{n}\right)=\mathrm{e}^{-x} \text { for } x \in \mathbb{R} .
$$

Then, as $n \rightarrow \infty$,

$$
\sum_{k=1}^{\infty} \varepsilon_{\left(k / n, a_{n}^{-1}\left(X_{k}-b_{n}\right)\right)} \stackrel{\mathrm{w}}{\rightarrow} \sum_{k=1}^{\infty} \varepsilon_{\left(s_{k}, P_{k}\right)} \quad \text { in } M_{P}([0, \infty) \times \mathbb{R}),
$$

where $\sum_{k=1}^{\infty} \varepsilon\left(s_{k}, P_{k}\right)$ is a $\operatorname{PRM}\left(\mathrm{d} t \times \mathrm{e}^{-x} \mathrm{~d} x\right)$.

Proof. First, we investigate the case in which $Z_{1} \in \mathcal{L}(\gamma)$ with $\gamma>0$. Let $u_{n}=a_{n} x+b_{n}$, $n \in \mathbb{N}$. Since $\left(X_{k}\right)_{k \in \mathbb{N}_{0}}$ is geometrically strongly mixing by Lemma 2.1 , the mixing condition $D_{r}\left(u_{n}\right)$ of [20, Theorem 5.5.1] holds for $\left(X_{k}\right)_{k \in \mathbb{N}_{0}}$. It remains to show the anti-cluster condition $D^{\prime}\left(u_{n}\right)$, i.e.

$$
\lim _{k \rightarrow \infty} \lim _{n \rightarrow \infty} n \sum_{j=2}^{\lfloor n / k\rfloor} \mathrm{P}\left(X_{j}>u_{n}, X_{1}>u_{n}\right)=0 .
$$

By Lemma 2.2 we have

$$
n \sum_{j=2}^{\lfloor n / k\rfloor} \mathrm{P}\left(X_{j}>u_{n}, X_{1}>u_{n}\right) \leq n \sum_{j=2}^{\lfloor n / k\rfloor} \mathrm{P}\left(\tilde{X}_{j}>u_{n}, \tilde{X}_{1}>u_{n}\right) .
$$

Furthermore, $\mathrm{P}\left(\tilde{X}_{1}>u_{n}\right) \sim C \mathrm{P}\left(X_{1}>u_{n}\right)$ as $n \rightarrow \infty$ and some constant $C>0$ by Proposition 4.1. Analogously to the proof of Theorem 7.4 of [26] (cf. [16, proof of Lemma 2]), the moving average process $\left(\tilde{X}_{k}\right)_{k \in \mathbb{N}_{0}}$ satisfies the $D^{\prime}\left(u_{n}\right)$ condition, and, hence, $\left(X_{k}\right)_{k \in \mathbb{N}_{0}}$ also satisfies the $D^{\prime}\left(u_{n}\right)$ condition. The conclusion then follows by [20, Theorem 5.5.1].

In the remaining case, $Z_{1} \in \delta \cap \operatorname{MDA}(\Lambda)$, the conclusion follows from [15, Proposition 9] and (4.3)-(4.4), which converges to 0 as $x \rightarrow \infty$.

We can now obtain the behavior of the running maxima of the stationary sequence and, hence, identify the extremal index to be equal to 1 , which implies that the model cannot exhibit clusters on high levels in this case. We omit the proof, which follows the lines of the proof of Corollary 3.1.

Corollary 4.1. Let the assumptions of Theorem 4.1 hold, and let $M_{n}=\max _{k=1, \ldots, n} X_{k}$ for $n \in \mathbb{N}$. Then

$$
\lim _{n \rightarrow \infty} \mathrm{P}\left(a_{n}^{-1}\left(M_{n}-b_{n}\right) \leq x\right)=\exp \left(-\mathrm{e}^{-x}\right) \text { for } x \in \mathbb{R} .
$$

In particular, the extremal index of $\left(X_{k}\right)_{k \in \mathbb{N}_{0}}$ is equal to 1 .

\section{Conclusion}

We have shown that stationary TAR models with noise in $\delta \cap \operatorname{MDA}(\Lambda)$ or $\mathcal{L}(\gamma)$ with $\gamma>0$ are tail equivalent to their noise sequence, and that they have extremal index equal to 1 ; hence, they cannot cluster. On the other hand, if the noise sequence is regularly varying then the tail 
is in general only O-regularly varying, but for $\operatorname{TAR}(S, 1)$ models with intervals as partitions, it is tail equivalent to its noise (in particular, it is regularly varying). Moreover, in this case the extremal index is less than 1 in many cases depending on the coefficients of the TAR model. In these cases the $\operatorname{TAR}(S, 1)$ model can exhibit cluster behavior.

It would be interesting to obtain similar results for noise sequences which are superexponential, such as distribution functions $F$ with tails like $\bar{F}(x)=K x^{b} \exp \left(-x^{p}\right)$ for $p \in(1, \infty)$. However, already the analysis for infinite moving average processes with such noise sequences is very involved and has been carried out by Rootzén [26], [27]. See also [19] for such moving average processes. But, for the TAR model, owing to the nonlinear regime switch, it seems an open problem how to determine the precise tail behavior of the stationary TAR model even for Gaussian noise, apart from simple situations such as symmetric TAR models.

\section{Acknowledgement}

The authors are indebted to a referee for pointing out the relation between $\mathcal{L}(\gamma)$ for $\gamma>0$ and $\operatorname{MDA}(\Lambda)$.

\section{References}

[1] AN, H. Z. AND HuAng, F. C. (1996). The geometric ergodicity of nonlinear autoregressive models. Statistica Sinica 6, 943-956.

[2] Basrak, B., Davis, R. A. And Mikosch, T. (2002). Regular variation of GARCH processes. Stoch. Process. Appl. 99, 95-115.

[3] Brachner, C. (2004). Tailverhalten autoregressiver Thresholdmodelle. Diploma Thesis, Technische Universität München.

[4] Breiman, L. (1965). On some limit theorems similar to the arc-sine law. Theory Prob. Appl. 10, 351-360.

[5] Chan, K. And Tong, H. (1985). On the use of the deterministic Lyapunov function for the ergodicity of stochastic difference equations. Adv. Appl. Prob. 17, 666-678.

[6] Chen, R. and Tsay, R. (1991). On the ergodicity of TAR(1) processes. Ann. Appl. Prob. 1, 613-634.

[7] Davis, R. A. AND Hsing, T. (1995). Point process and partial sum convergence for weakly dependent random variables with infinite variance. Ann. Prob. 23, 879-917.

[8] DAVIS, R. AND RESNICK, S. (1985). Limit theory for moving averages of random variables with regularly varying tail probabilities. Ann. Prob. 13, 179-195.

[9] Davis, R. AND Resnick, S. (1988). Extremes of moving averages of random variables from the domain of attraction of the double exponential distribution. Stoch. Process. Appl. 30, 41-68.

[10] Diop, A. And Guegan, D. (2004). Tail behavior of a threshold autoregressive stochastic volatility model. Extremes 7, 367-375.

[11] Embrechts, P., Goldie, C. M. and Veraverbeke, N. (1979). Subexponentiality and infinite divisibility. Z. Wahrscheinlichkeitsth. 49, 335-347.

[12] Embrechts, P., Klüppelberg, C. And Mikosch, T. (1997). Modelling Extremal Events for Insurance and Finance. Springer, Berlin.

[13] FAn, J. AND YaO, Q. (2003). Nonlinear Time Series: Nonparametric and Parametric Methods. Springer, New York.

[14] FASEN, V. (2005). Extremes of regularly varying Lévy-driven mixed moving average processes. Adv. Appl. Prob. 37, 993-1014.

[15] Fasen, V. (2006). Extremes of subexponential Lévy driven moving average processes. Stoch. Process. Appl. 116, 1066-1087.

[16] Fasen, V., KlüPpelberg, C. And Lindner, A. (2006). Extremal behavior of stochastic volatility models. In Stochastic Finance, eds A. Shiryaev et al., Springer, New York, pp. 107-155.

[17] Feller, W. (1971). An Introduction to Probability Theory and Its Applications, Vol. II, 2nd edn. John Wiley, New York.

[18] Hsing, T. (1993). On some estimates based on sample behavior near high level excursions. Prob. Theory Relat. Fields 95, 331-356.

[19] KlüPPElBerg, C. And Lindner, A. (2005). Extreme value theory for moving average processes with light-tailed innovations. Bernoulli 11, 381-410.

[20] Leadbetter, M. R., Lindgren, G. and Rootzén, H. (1983). Extremes and Related Properties of Random Sequences and Processes. Springer, New York. 
[21] Meyn, S. And Tweedie, R. (1993). Markov Chains and Stochastic Stability. Springer, London.

[22] Pakes, A. G. (2004). Convolution equivalence and infinite divisibility. J. Appl. Prob. 41, 407-424. (Correction: 44 (2007), 295-305.)

[23] ReSNick, S. I. (1986). Point processes, regular variation and weak convergence. Adv. Appl. Prob. 18, 66-138.

[24] Resnick, S. I. (1987). Extreme Values, Regular Variation, and Point Processes. Springer, New York.

[25] Resnick, S. I. (2007). Heavy-Tail Phenomena. Springer, New York.

[26] Rootzén, H. (1986). Extreme value theory for moving average processes. Ann. Prob. 14, 612-652.

[27] Rootzén, H. (1987). A ratio limit theorem for the tails of weighted sums. Ann. Prob. 15, 728-747.

[28] Tong, H. (1977). Contribution to the discussion of the paper entitled 'Stochastic modelling of riverflow time series' by A. J. Lawrance and N. T. Kottegoda. J. R. Statist. Soc. A 140, 34-35.

[29] Tong, H. (1990). Nonlinear Time Series. Oxford University Press.

[30] Tong, H. And Lim, K. S. (1980). Threshold autoregression, limit cycles and cyclical data. J. R. Statist. Soc. B 42, 245-292. 